\title{
Caccia all'arte. Ideologia, potere, razzie e conseguenze di una smisurata passione nazista
}

\author{
di Elena Pirazzoli
}

Giu 12, $2019 \mid \underline{\text { In evidenza, }}$ Storia pubblica $\mid \underline{0 \mid}$

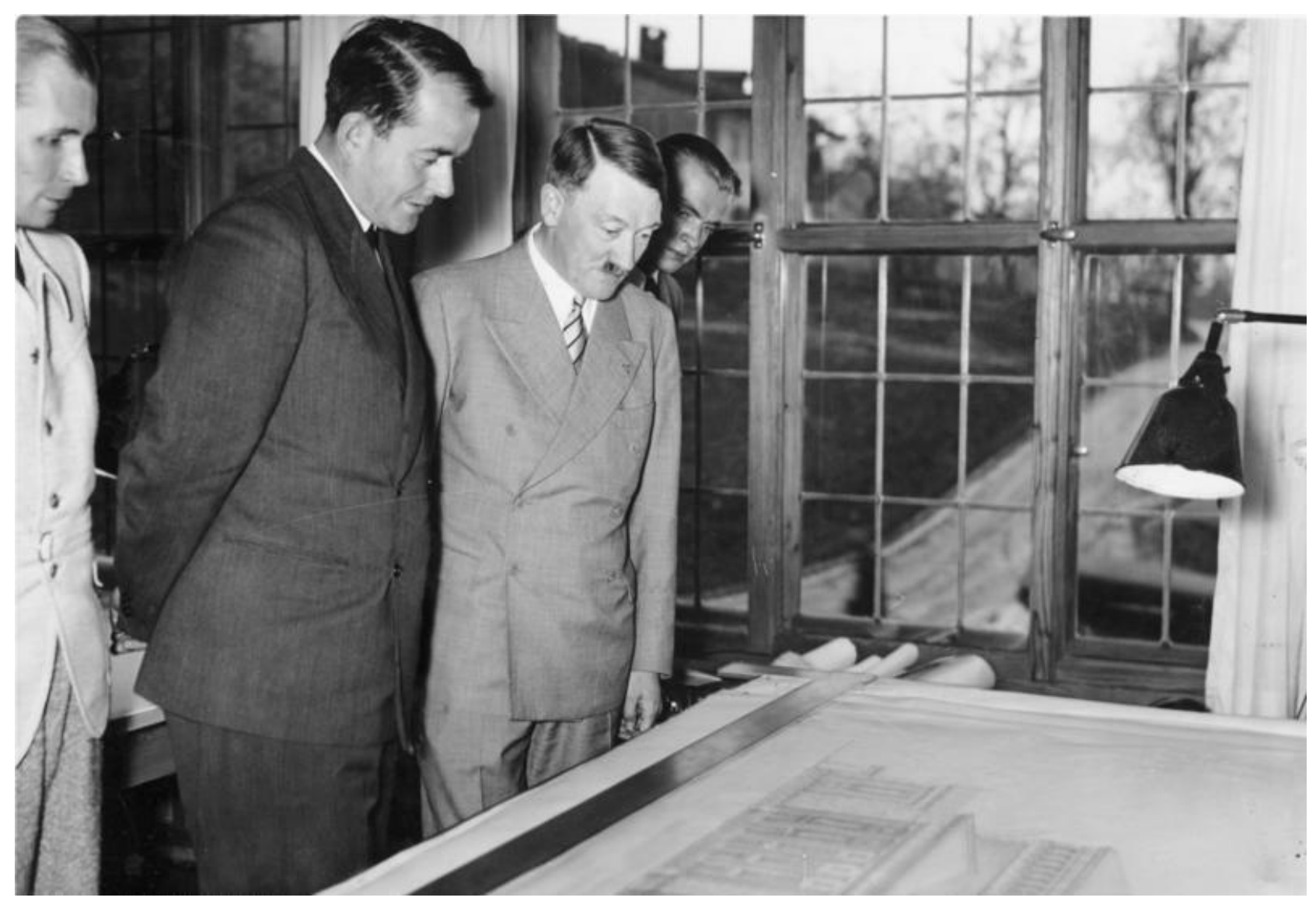

Adolf Hitler con Albert Speer al Berghof sull'Obersalzberg davanti ai progetti della nuova Opernhaus per Linz (Bundesarchiv, Bild 183-2004-1103-500 / CC-BY-SA 3.0)

\section{Abstract}

Il rapporto tra nazismo e arte è molto stretto: il regime si adoperò per normare il settore artistico in modo che stile e contenuto fossero conformi alla sua visione politica, sociale, razziale. L'articolo riflette in particolare sulla Kunstraub, l'arte "razziata" dai nazisti attraverso un minuzioso programma di censimento, espropriazione e inventariazione di opere d'arte, soprattutto a danno degli ebrei. Per attuare questo ampio e articolato piano estetico e culturale, vennero mobilitati architetti, ma anche storici dell'arte, mercanti ed esperti d'arte e antiquariato. Il nome oggi più noto è quello di Hildebrand Gurlitt, la cui "collezione" è riemersa solo di recente, grazie a un fortuito controllo effettuato nei confronti di suo figlio Cornelius nel 2012, ritrovando 1.500 opere di provenienza dubbia, connessa con le razzie naziste. Alla morte di Cornelius Gurlitt, le opere sono state ereditate dal Kunstmuseum di Berna, che si è impegnato a conservarle in attesa del ritrovamento dei discendenti delle persone cui furono sottratte. Simili progetti sono oggi frequenti (celebre il caso di Maria Eichhorn), ma nonostante le numerose battaglie - anche legali -, risalire ai 
legittimi proprietari o ai loro eredi è complesso, perché il loro destino è quasi sempre lo stesso: la morte nei campi di sterminio.

\section{La recente fortuna cinematografica del tema}

La razzia delle opere d'arte messa in atto in modo sistematico dal regime nazista in tutti i Paesi occupati durante il secondo conflitto mondiale è un tema che suscita grande interesse anche in ambito non specialistico. Probabilmente il fascino dei salvataggi rocamboleschi, lo stagliarsi di figure "eroiche" e un tema in apparenza meno greve (benché strettamente connesso con la deportazione e lo sterminio, come vedremo) rendono questo aspetto del regime nazista, dell'occupazione e della guerra più accessibile e "sostenibile" a un pubblico ampio. Non a caso negli ultimi anni diversi film si sono focalizzati su questo tema: The Monuments Men (George Clooney, USA/Germania 2014), classico film di guerra in cui il conflitto viene visto dalla prospettiva di una squadra di storici dell'arte, dell'architettura e restauratori, impegnati nel salvataggio delle opere razziate dai nazisti; Woman in Gold (Simon Curtis, GB 2015), concepito come un legal drama attorno alla restituzione del ritratto di Adele Bloch-Bauer a firma di Gustav Klimt all'erede della famiglia di ebrei austriaci cui venne sottratto, contrapposta alla Galleria del Belvedere di Vienna che lo aveva incamerato nel 1938; il sofisticato Francofonia (Aleksandr Sokurov, Francia/Germania/Olanda 2015), che racconta la relazione di opposizione e poi di collaborazione tra Jacques Jaujard, conservatore del Louvre durante l'occupazione della Francia e il conte Franziskus Wolff-Metternich, inviato da Berlino per valutare il patrimonio artistico del museo parigino e trasferirne parte in Germania, che invece aiutò lo storico dell'arte francese a proteggere le opere; infine, il documentario Hitler contro Picasso e gli altri (Claudio Poli, Italia/Francia/Germania 2018), il cui titolo non rende la complessità di intreccio che il film ben affronta, mostrando la strettissima connessione tra deportazione degli ebrei, spoliazione dei loro beni e creazione delle collezioni di Hitler e Göring. Anche solo passando in rassegna questi pochi titoli emergono alcuni tratti fondamentali della relazione tra nazismo e arte, fino alle conseguenze post belliche di tale passione smisurata e morbosa. In questo articolo si cercheranno di mettere in luce le motivazioni sottese alla spoliazione del patrimonio artistico europeo - pubblico e privato - e le modalità con cui questa si è data, i tentativi di resistenza per arginare le razzie e le forme di restituzione delle opere alla conclusione del conflitto. Ma la questione della NS-Raubkunst - il saccheggio nazista dell'arte - è molto forte ancora oggi, rendendo la Provenienzforschung, la ricerca della provenienza, un importante ambito degli studi delle collezioni museali internazionali, spesso attivato dagli stessi musei, con complessi risvolti legali. 


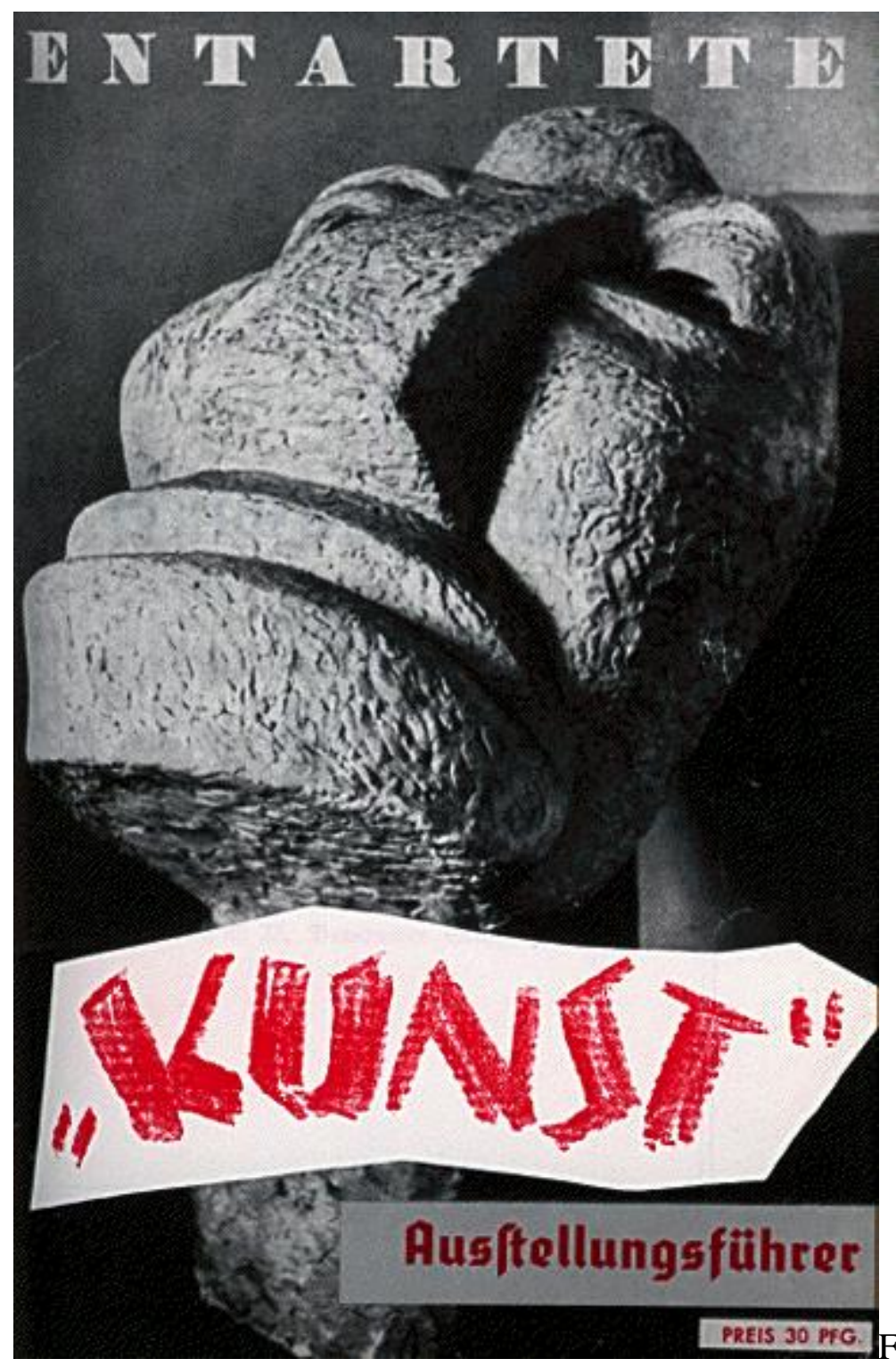

Kunst, Monaco, 1937. La scultura è Grande Tête di Otto Freundlich del 1912, ribattezzata sarcasticamente dai nazisti Der neue Mensch ("l'uomo nuovo")[1]. 


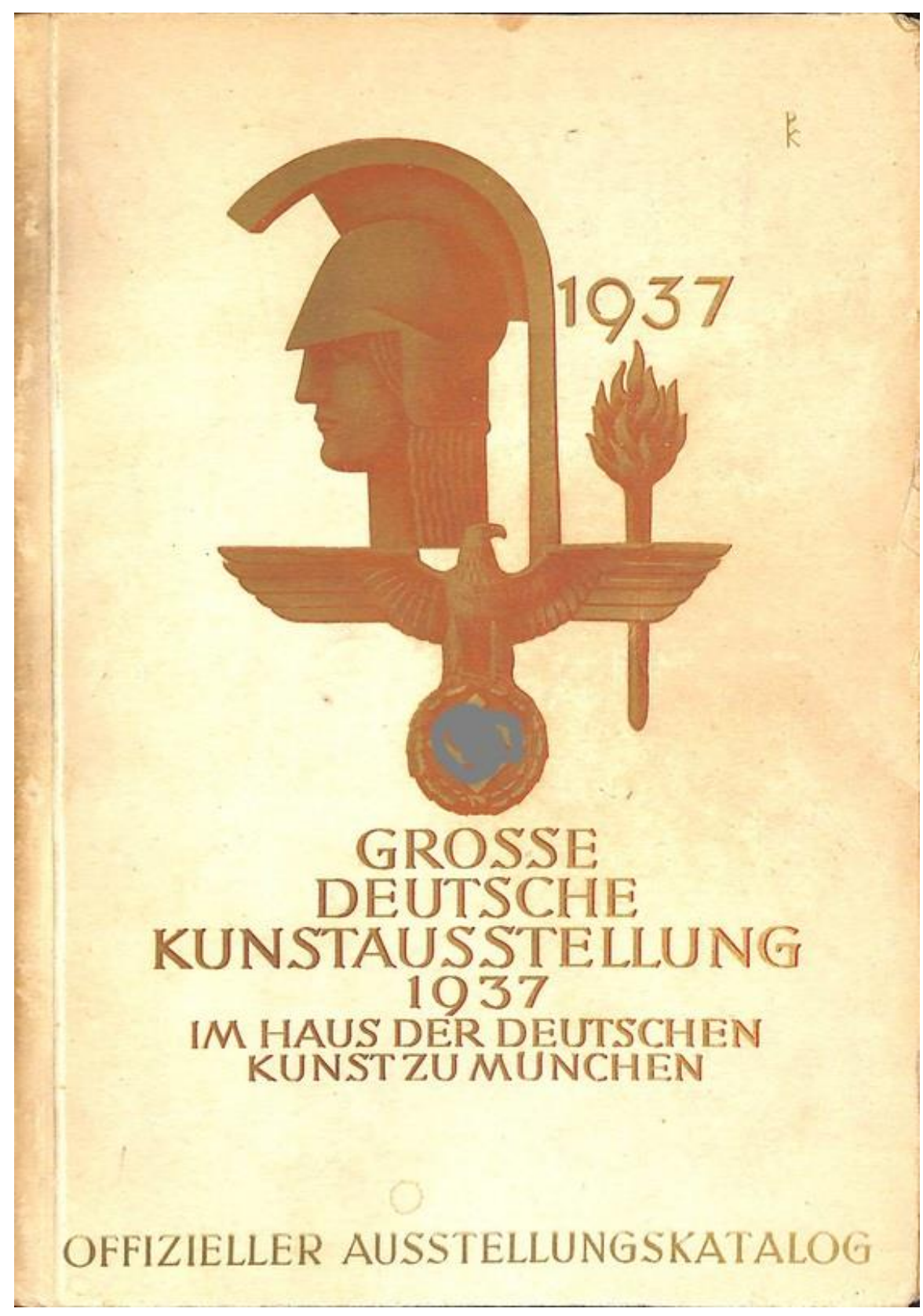

Fig. 2 Copertina del catalogo della prima Grosse Deutsche Kunstausstellung, Monaco, 1937

\section{Il nazismo e il controllo dell'arte}

Il nazismo, infatti, ebbe fin dalla presa del potere un forte interesse per il controllo dell'arte: la necessità di veicolare l'ideologia e i temi fondamentali del regime non si limitava alla supervisione dei contenuti, ma anche delle forme. Tutto quello che non rispondeva ai canoni di purezza germanica venne schernito, bollato come "degenerato"[2], separato ed espunto dal novero dell'autentica arte tedesca, o meglio, della visione nazista dell'arte tedesca[3]. Il 19 luglio 1937 inaugurò a Monaco, nell'Hofgarten, la nota mostra Entartete Kunst che raccoglieva 650 opere "non conformi”, requisite ed epurate dalle collezioni dei musei tedeschi. Impressionismo, dadaismo, espressionismo, Neue Sachlichkeit, surrealismo, cubismo e fauvismo: le principali correnti artistiche del primo Novecento erano rappresentate in questa mostra in negativo - una anti-mostra, allestita appositamente in modo caotico - che, dopo Monaco, venne portata in dodici città, terminando il suo percorso nel 1941. Si stima che tre milioni di visitatori videro quelle opere che, probabilmente, senza questa esposizione "al pubblico ludibrio" sarebbero rimaste appannaggio di 
circoli ristretti di collezionisti, artisti, galleristi, appassionati. Per paradosso, la mostra dell'Entartete Kunst esibiva agli occhi della massa dei cittadini tedeschi quello che si definiva in quell'occasione inguardabile e, di conseguenza, veniva bandito dal nuovo Reich.

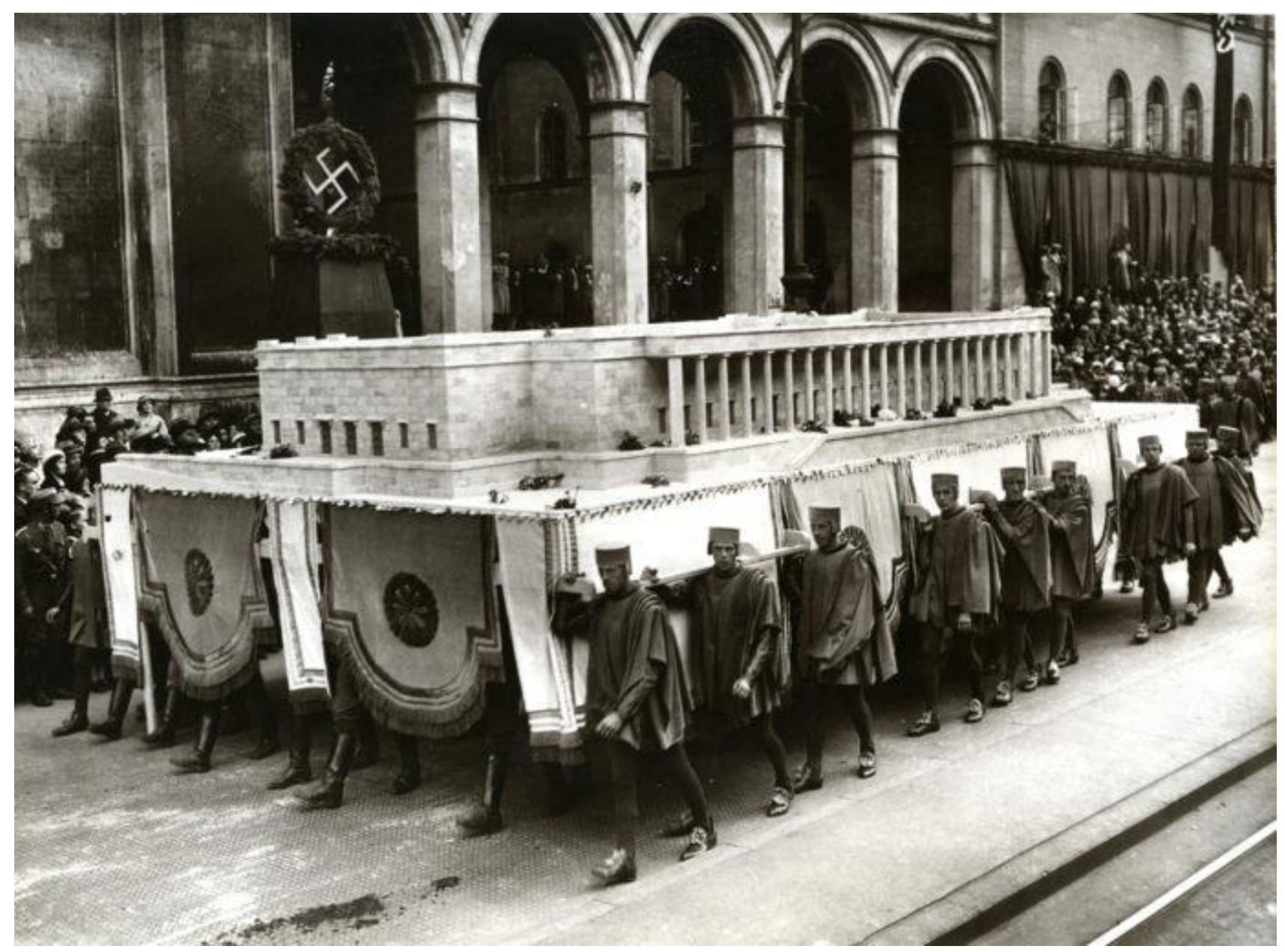

Fig. 3 Corteo per il Tag der Deutschen Kunst, Monaco, 1933. Sfila il modello della futura Haus der Deutschen Kunst.

Meno ricordata oggi è la mostra che aprì il giorno precedente, sempre a Monaco, nel nuovo museo inaugurato contestualmente - progettato da Paul Ludwig Troost per volontà di Hitler subito dopo la presa del potere - ovvero l'Haus der Deutschen Kunst. Die Grosse Deutsche Kunstausstellung era dedicata alla "grande arte tedesca", caratterizzata da forme neoclassiciste e allo stesso tempo nordiche, venate di elementi rustici e vernacolari (si pensi alla Bäuerliche Venus, la "venere contadina" del pittore bavarese Sepp Hilz o alla sua Bäuerliche Trilogie, "trilogia contadina", presentata in una delle edizioni dell 'esposizione, e fiera d'arte, monacense, che si tenne fino al 1944)[4]. Come scrive Hans Belting, "a Monaco nel 1937 si fronteggiarono due mostre in cui si separava l'arte 'malata' o 'degenerata' dall'arte 'sana' o ' tedesca' e in cui si distinguevano perciò in maniera netta ed evidente il male e il bene nell' arte" [5]. L'autentica arte tedesca avrebbe dovuto essere, da quel momento in poi, rappresentata da quegli artisti e quelle opere capaci di rievocare gli elementi fondanti della tradizione germanica - la natura, il Medioevo, il mondo rurale, la celebrazione dell'Heimat, la "patria" - impastandoli con l'ideologia razzista di supremazia ariana e con l'affermazione nazionalista, fino a creare un'arte atemporale e, quindi, eterna. 


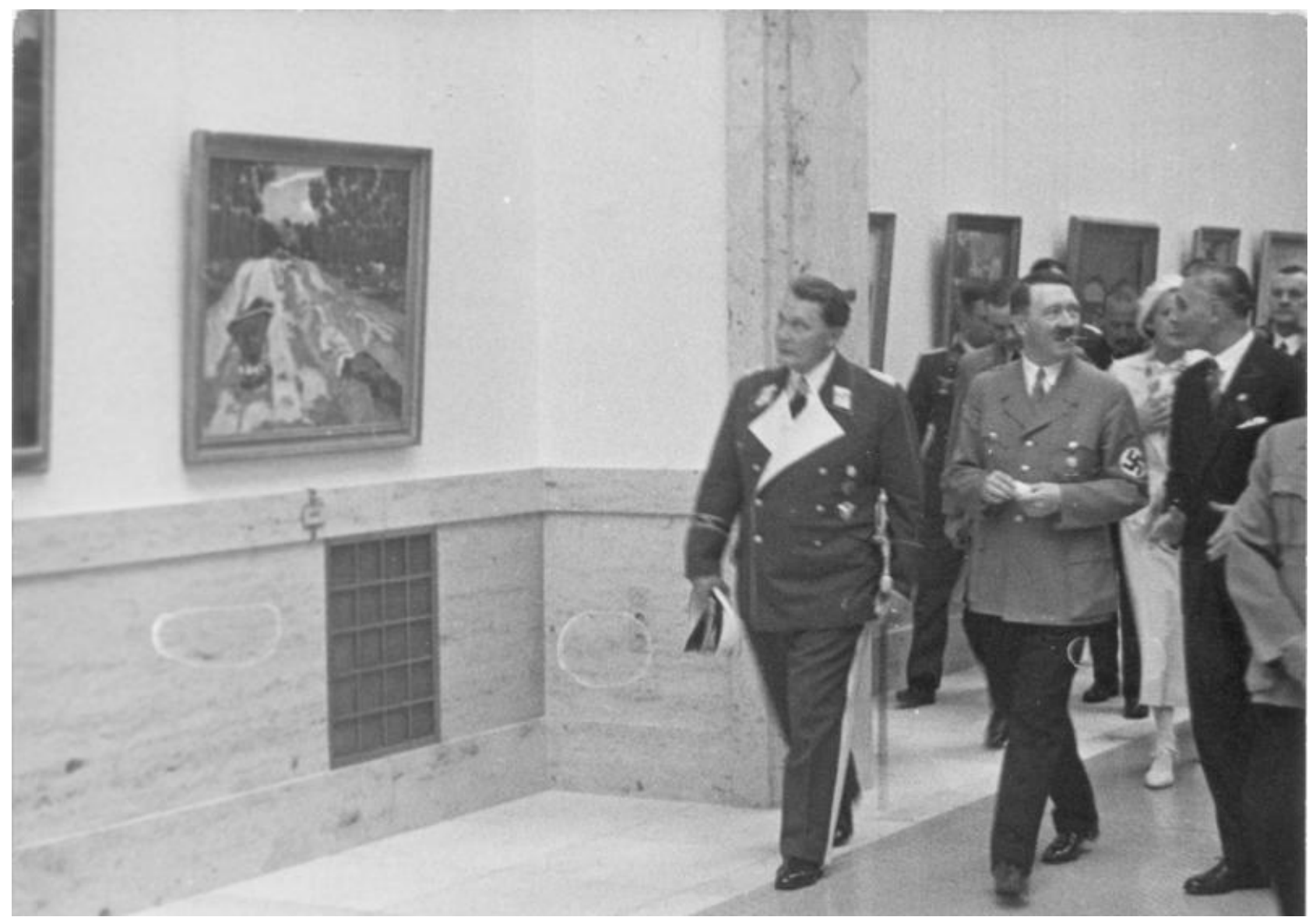

Fig. 4 Hitler und Göring im Haus der Deutschen Kunst - By Bundesarchiv, Bild 183-C10110 / CCBY-SA 3.0, CC BY-SA 3.0 de, $\underline{\text { Link }}$

\section{Hitler e il potere dell'estetica}

È in particolare il Führer ad avere un legame speciale, ossessivo, con l'arte. Un aspetto sottolineato con sarcasmo già dagli oppositori del regime come Bertolt Brecht, che dileggiava la sua pittura mediocre chiamandolo der Anstreicher, 1' "imbianchino"; o come Thomas Mann, che dall'esilio americano ne analizzava "il carattere e il destino" incentrandosi sulla definizione che Hitler stesso prediligeva per sé, ovvero quella di artista, benché respinto più volte alla selezione per entrare all'Accademia di Belle Arti di Vienna: artista incompreso, che ha covato risentimento e vendetta, sposandoli poi al sentimento nazionalista tedesco frustrato dopo la conclusione della Grande guerra[6]. L'ossessione per l'arte di Hitler è stata a lungo sottovalutata come aspetto marginale e per certi aspetti caricaturale. Agli inizi degli anni Duemila, lo storico della cultura Frederic Spotts ha invece assunto questa prospettiva per analizzare l'affermazione del nazismo[7], mettendo in luce come l'arte e la cultura - rifondate radicalmente e plasmate dall'ideologia del regime - avevano avuto il ruolo di strumenti per la conquista e il consolidamento del potere. I grandi apparati scenografici per le adunate, le architetture da megalomani (secondo una definizione dello stesso Albert Speer[8]), i nuovi musei e le esposizioni: tutto doveva funzionare come dispositivo di formazione e acculturazione delle masse dei nuovi sudditi del Reich. In particolare, cinque città, le principali del nuovo Reich, avrebbero dovuto essere trasformate e plasmate secondo questa nuova visione estetica. Le Führerstädte - le "città del Führer" - coinvolte nel progetto erano Berlino, futura Welthauptstadt Germania, capitale del Reich (e, in prospettiva, del mondo, "die Welt"), Norimberga, città del Parteitag, il raduno del partito, Monaco, Hauptstadt der Bewegung in quanto culla del movimento nazista, Amburgo, il porto principale, e infine Linz, la città della giovinezza di Hitler (a pochi chilometri da Branau, il suo paese natale), che sarebbe diventata così imponente da oscurare Vienna e Budapest, le due città degli splendori asburgici. 


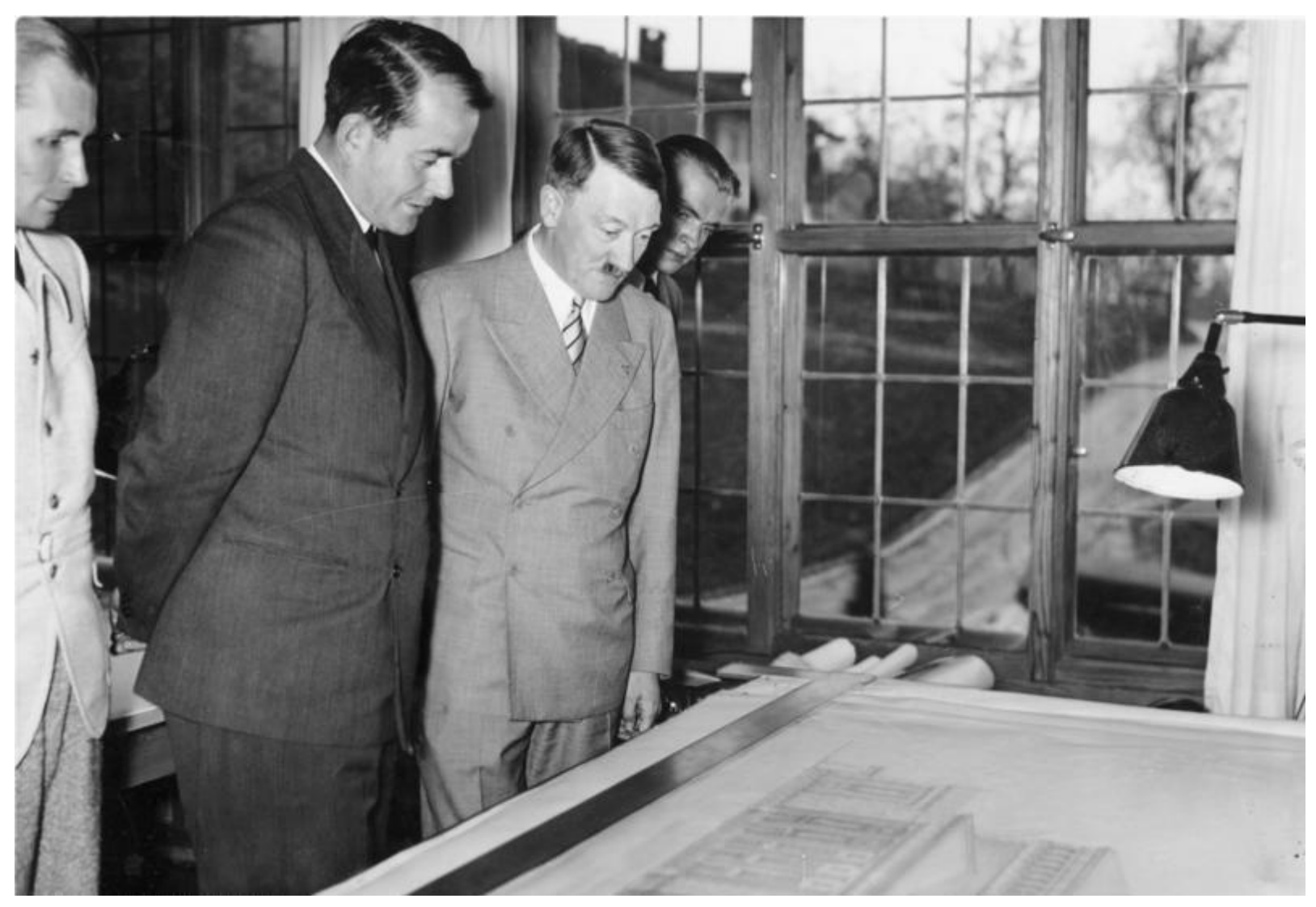

Fig. 5 Il Führer con Albert Speer al Berghof sull'Obersalzberg davanti ai progetti della nuova Opernhaus per Linz (Bundesarchiv, Bild 183-2004-1103-500 / CC-BY-SA 3.0)

\section{La guerra e la spoliazione dei Paesi occupati}

A partire dall'Anschluss dell'Austria nel marzo del 1938, a questo progetto "estetico" del regime si affiancarono operazioni di spoliazione delle collezioni d'arte - in particolare quelle private - dei Paesi occupati dalla Germania nazista. All'Austria seguì la Cecoslovacchia poi, dopo lo scoppio del conflitto, Polonia, Paesi Bassi, Francia. Dopo 1'8 settembre 1943, anche l'Italia venne coinvolta nelle razzie di collezioni private e pubbliche, condotte spesso sotto le mentite spoglie della tutela, affidata al Kunstschutz, il servizio militare di protezione delle opere dai bombardamenti.[9] Furono in particolare due collezioni a essere implementate attraverso vendite forzate e confische: quella che Hitler voleva mettere insieme per il Führermuseum da realizzare a Linz e la raccolta personale di Hermann Göring[10]. Questo doppio, spasmodico e vorace interesse generò una sistematica razzia di opere d'arte cui non scamparono soprattutto le collezioni di famiglie ebraiche (dei Rotschild, di Rudolf Gutmann, Fritz Mannheimer, Alphonse Kann, Adolphe Schloss e moltissimi altri) e oppositori del regime (ad esempio, Fritz Thyssen). L'intermediazione di mercanti d'arte dei diversi paesi fu fondamentale: un'ampia rete di figure in relazione e in concorrenza l'una con l'altra. In alcuni casi si trattò anche di mercanti di origine ebraica, motivati ancora più degli altri a mostrare le proprie capacità nel trovare opere, per ottenere in cambio protezione e visti, evitando un destino di deportazione e morte[11]. Vennero così scelti esperti d'arte (studiosi e direttori di musei) incaricati di individuare le opere e acquisirle (grazie alla dotazione di fondi illimitati per poter adempiere al compito) ma anche, dopo lo scoppio della guerra, furono creati dei commandi speciali che propendevano per metodi più sbrigativi: confische e saccheggi. In questa fase le "prede" del vorace interesse degli occupanti non furono solo dipinti e sculture, monete, medaglie e armature storiche, ma anche libri, di pregio e non, arredi, strumenti musicali, orologi, tessuti, fino addirittura alle stoviglie. 
Nel 1939 venne creata la Sonderauftrag Linz, una missione speciale con il compito di individuare dipinti e sculture che avrebbero dovuto fare parte del Führermuseum, il museo che Hitler voleva realizzare a Linz. Un museo grandioso, con una collezione pensata direttamente dal Führer: presentata ufficialmente, per legittimare lo sforzo economico, come un dono ai tedeschi da parte del loro capo, era in realtà un monumento a se stesso, una soddisfazione del proprio desiderio sia di possesso che di plasmazione del gusto estetico a venire. Già nel 1925 Hitler aveva disegnato nel suo album una bozza di un museo tedesco per Berlino (Nationalgalerie), non limitandosi al progetto per l'edificio ma ipotizzando anche la rosa degli autori in collezione e la suddivisione delle loro opere nelle sale. Nel 1933, subito dopo la presa del potere, aveva immediatamente commissionato a Troost, allora il suo architetto prediletto[12], l'Haus der Deutschen Kunst di Monaco, ma dopo l'Anschluss il progetto cambiò: il nuovo museo avrebbe dovuto dare rilievo e preminenza nel nuovo Reich alla città della sua giovinezza, contrastando e sovrastando Vienna, la cui Accademia delle Belle Arti lo aveva sempre escluso[13]. Per realizzare il suo museo, Hitler si rivolse all'architetto Roderich Fick, al mercante d'arte di Berlino Karl Haberstock e soprattutto ad Hans Posse, direttore della Pinacoteca di Dresda[14], cui diede mandato assoluto e fondi illimitati per mettere insieme la raccolta delle migliori opere dell'arte europea. Ma questo non sarebbe bastato se non si fosse ampliato il bacino cui poter attingere: l'annessione dell'Austria prima, l'invasione della Cecoslovacchia e poi, con lo scoppio della guerra, della Polonia, dei Paesi Bassi e della Francia generarono le condizioni che resero possibile la creazione di un mercato dell'arte fiorente e disperato. In certi casi le opere vennero acquisite tramite pagamento, ma bisogna considerare in quali condizioni si svolsero le compravendite: molti collezionisti, facoltosi imprenditori di origine ebraica, vennero costretti a vendere sotto minaccia oppure con la promessa (spesso rivelatasi falsa) di poter così ottenere un visto. Se invece si trattava di collezionisti già fuggiti, imprigionati come oppositori del regime, o deportati nei campi, le loro proprietà vennero confiscate dal Reich. Benché la Convenzione dell'Aja del 1907 avesse stabilito la tutela della proprietà privata in caso di guerra, nel caso di ebrei, oppositori e fuggitivi non venne applicata, perché considerati "nemici pubblici”[15]. Il contesto bellico, inoltre, favorì il saccheggio e i veri e propri furti. I gusti in materia d'arte del Führer erano molto tradizionalisti, amava in particolare i pittori tedeschi del romanticismo, attivi nell'Ottocento a Vienna e a Monaco. Ma Hans Posse prese molto sul serio il compito di realizzare il museo con la migliore arte europea dalle origini fino al primo Novecento, contrastando e contenendo le predilezioni di Hitler, e indirizzandolo anche verso la pittura fiamminga e del Rinascimento italiano. 


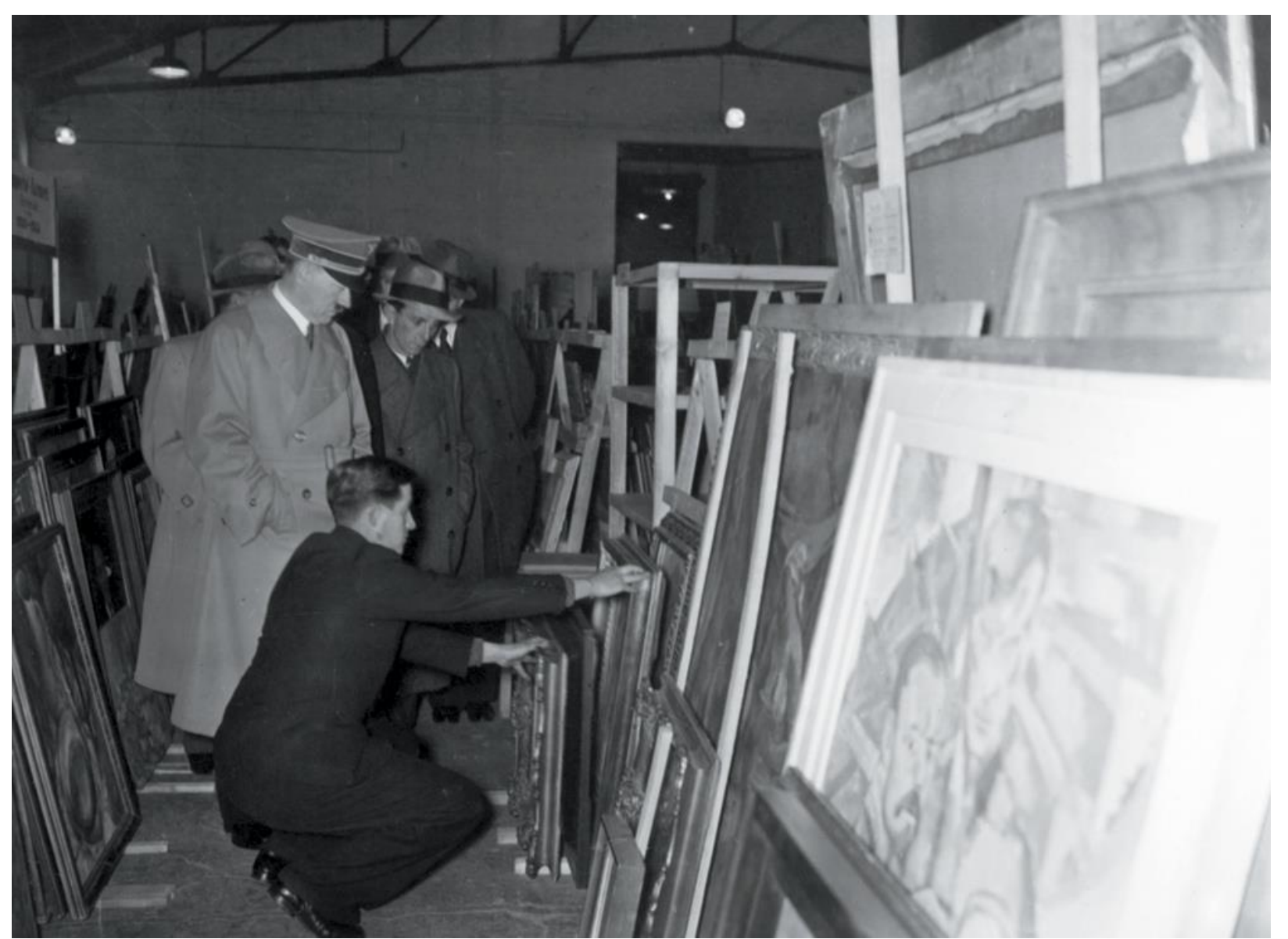

Fig. 6 Hitler, insieme a Goebbels, esamina dipinti stoccati in un deposito (National Archives, Stati Uniti).

\section{Carinhall, una reggia per la collezione Göring}

Anche il numero due del regime nazista, il maresciallo Hermann Göring, era affetto dalla stessa morbosa passione per l'arte. A differenza di Hitler, Göring poteva vantare origini aristocratiche, ma fu soprattutto la crescita del suo potere tramite il nazismo a portarlo ad atteggiarsi a principe rinascimentale, circondato di sfarzo, opulenza e arte. La sua reggia divenne il castello di Carinhall, in Brandeburgo, dedicato alla prima moglie, una nobildonna svedese morta precocemente. Nelle sue stanze, Göring riceveva il Führer e gli altri vertici del regime, come anche ambasciatori e capi di Stato, esibendo i suoi trofei di caccia: teste di cervo e opere d'arte. Inizialmente acquistò alcuni dipinti del Rinascimento italiano e del Cinquecento tedesco presso un'importante galleria romana, cui si aggiunsero altre opere ottenute tramite l'intermediazione di Walter Hofer[16], un mercante d'arte di Monaco; con lo scoppio del conflitto, però, la sua collezione si incrementò in modo più spregiudicato. Dopo il 1940, nei Paesi Bassi e in Francia Göring fece soprattutto incetta di opere di galleristi e collezionisti ebrei: i loro beni venivano "legalmente" confiscati dagli uomini del maresciallo in quanto proprietà di nemici dello Stato, per questo posti sotto tutela e requisiti. Dalle raccolte del gallerista parigino Paul Rosenberg o del mercante d'arte olandese Jacques Goudstikker il maresciallo del Reich si accaparrò dipinti di Rembrandt, Van Dyck, Cranach, Poussin, Fragonard, Velázsquez, Goya, Veronese, Tintoretto, Canaletto, ma anche opere ufficialmente bandite, di autori moderni "degenerati", come Monet, Renoir, Cézanne[17]. Una delle figure più attive nell'opera di confische fu Bruno Lohse[18], laureato in storia dell'arte, mercante d'arte, membro del partito nazista e delle SS. Nel 1940 Lohse divenne uno degli uomini chiave a Parigi dell'Einsatzstab Reichsleiter Rosenberg (ERR), commando preposto alle confische: il compito di Lohse era quello di selezionare tra i beni sequestrati opere per il Führermuseum, ma in parallelo agiva per conto di Göring, inserendosi così nella competizione tra i due prominenti del nazismo. Tutti questi dipinti e 
sculture venivano stoccati in depositi e poi inviati in Germania. A Parigi, il principale luogo di raccolta divenne prima una sezione del Louvre, poi il museo dello Jeu de Paume: tra il 1940 e il 1942 al suo interno vennero organizzate dall'ERR delle vere e proprie esposizioni "private", soprattutto per Hermann Göring, dove il maresciallo, unico privilegiato visitatore, poteva scegliere le opere di suo gusto da trasferire a Carinhall[19]. La gerarchia prevedeva che il Führer avesse la precedenza nella scelta, secondo veniva Göring, in terza battuta altre figure di spicco del regime, in quarta in musei tedeschi, infine, la vendita sul mercato estero, in particolare presentando i lotti nelle case d'asta svizzere (destino previsto soprattutto per le opere di arte "degenerata")[20]. Tuttavia, contro questa movimentazione di opere d'arte si attivarono azioni di resistenza: in particolare Rose Valland, conservatrice dello Jeu de Paume, in accordo con il direttore dei Musées nationaux Jacques Jaujard, iniziò ad annotare il passaggio delle opere, la provenienza e i luoghi di destinazione, scattando anche fotografie. Un lavoro prezioso e rischioso, a partire da cui la storica dell'arte iniziò, subito dopo la liberazione di Parigi nell'ottobre 1944, un capillare ricerca dei beni trafugati, collaborando con le commissioni degli Alleati[21].

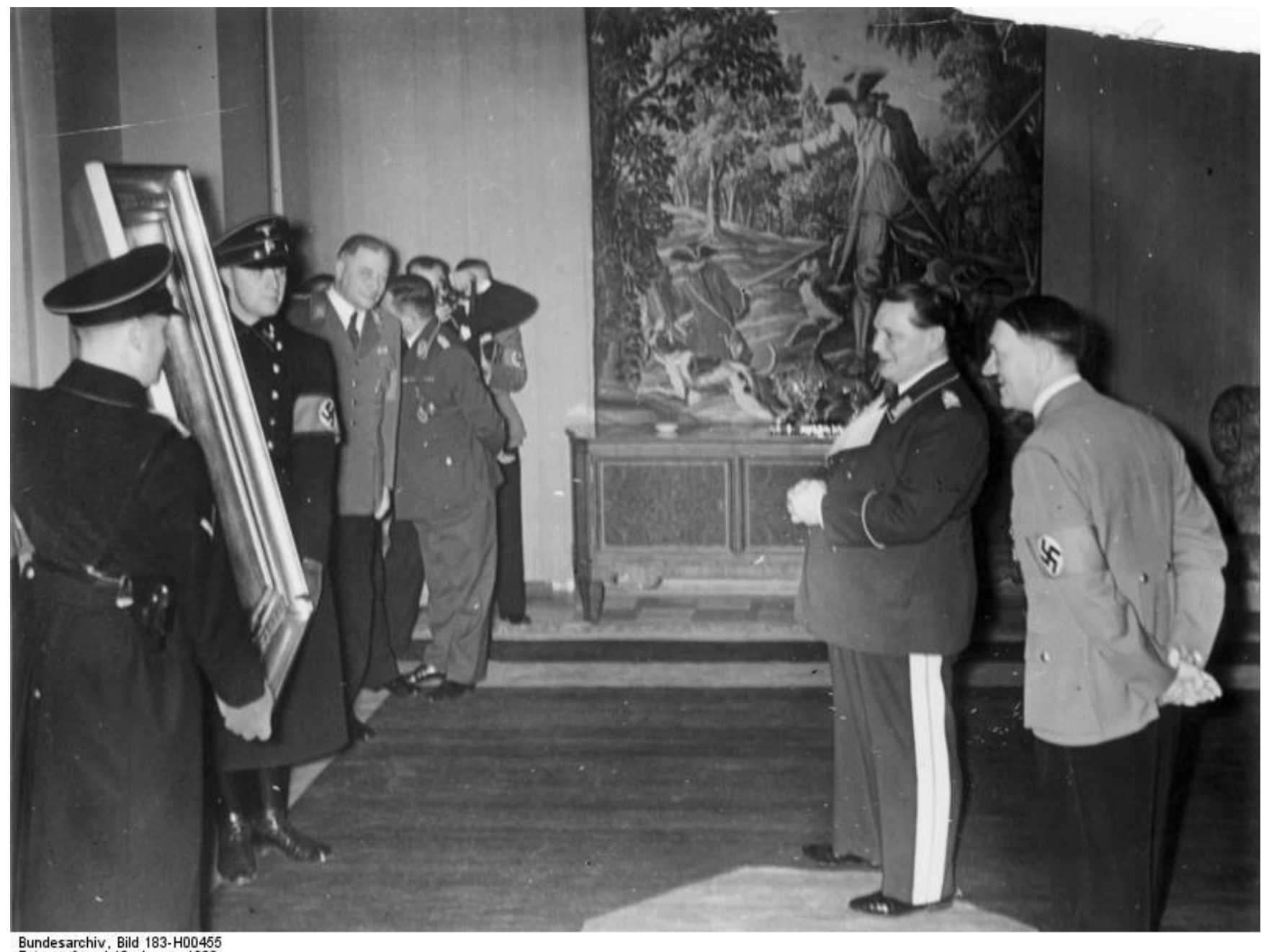
Foto: 0. Ang. | 12. Januar 1938

Fig. 7 Adolf Hitler regala un dipinto a Hermann Göring per il suo compleanno, 12 gennaio 1938 (Bundesarchiv, Bild 183-H00455 / CC-BY-SA 3.0)

\section{L'Einsatzstab Reichsleiter Rosenberg (ERR) e i beni ebraici}

L'Einsatzstab Reichsleiter Rosenberg (ERR) venne creato nel luglio del 1940: una squadra di intervento sotto il comando di Alfred Rosenberg, uno dei principali ideologi del nazismo, che mise in atto la spoliazione sistematica dei beni degli ebrei nei Paesi occupati. Questi materiali dovevano servire alla Hohe Schule der NSDAP[22], una scuola di perfezionamento per l'élite del partito 
concepita da Rosenberg: per capire i nemici del nazismo era necessario studiare gli oggetti della loro cultura. La Hohe Schule doveva articolarsi in diversi istituti (di studi biologici e razziali, studi coloniali, indogermanici, religiosi, ecc.) sparsi in diverse città del Reich. Uno dei pochi a entrare già in attività prima della guerra fu l'Institut zur Erforschung der Judenfrage, 1' "istituto per la ricerca della questione ebraica" con sede a Francoforte. Furono in particolare i libri ebraici, dai rotoli della Torah ai codici miniati, come anche gli oggetti rituali e gli arredi sacri, a essere confiscati e inviati in Germania. Il territorio principale di queste razzie fu l'est Europa, dove si trovavano le comunità più numerose: Polonia, Lituania, Galizia, Boemia[23]. L'ERR fu molto attivo anche a Parigi, in particolare nell'articolazione del Dienststelle Westen (il "servizio occidentale"), dove arrivò a prelevare dalle case degli ebrei - deportati nei campi di concentramento e di sterminio - oggetti di ogni tipo: la Möbel Aktion fu una sistematica razzia di mobili, porcellane, tappeti, pianoforti, libri, orologi, stoviglie e giocattoli, ecc. Nei suoi depositi nei sotterranei del Musée d'Art Moderne in rue de Tokyo, nell'ex grande magazzino Lévitan, in alcuni annessi della Gare d'Austerlitz e in uno stabile in rue Bassano, venne catalogato tutto in modo minuzioso, suddividendo le tipologie degli oggetti prelevati, grazie al lavoro effettuato dalla manodopera di prigionieri ebrei provenienti dal campo di concentramento di Drancy, nella periferia nordest della capitale[24]. Gli oggetti requisiti dovevano essere mandati nelle zone bombardate della Germania e nei territori conquistati in Europa orientale, dal 1941 sotto l'amministrazione proprio di Rosenberg, a capo del Reichsministerium für die besetzten Ostgebiete (il ministero del Reich per i territori occupati a est).

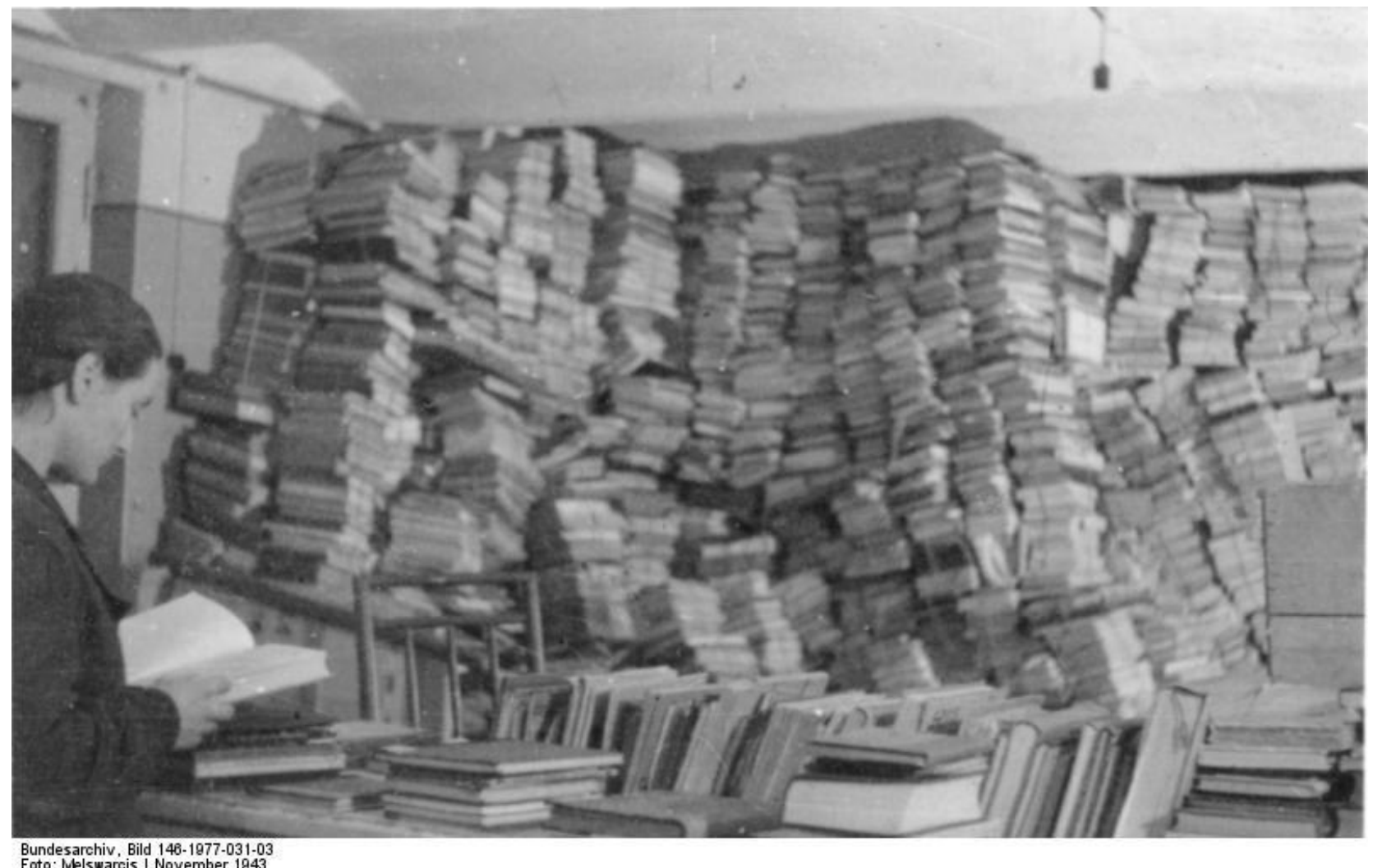

Fig. 8 Deposito di libri nel seminterrato del Dienststelle ERR, Riga, november 1943 (Bundesarchiv, Bild 146-1977-031-03 / Melswarcis / CC-BY-SA 3.0)

Alla fine di settembre del 1943 due ufficiali dell'ERR, Johannes Pohl[25] e Hans Grunewald, si presentarono anche agli uffici della comunità ebraica di Roma, presso la sinagoga. Al piano superiore si trovavano raccolti i pregiati volumi della Biblioteca della Comunità israelitica della capitale e della Biblioteca del Collegio rabbinico italiano, trasferita da Firenze. L'inizio della razzia avvenne l'11 ottobre, per protrarsi fino a dicembre. Mentre gli abitanti del ghetto di Roma venivano deportati verso Auschwitz, i circa 25.000 volumi della loro comunità furono inviati in Germania: a 
tutt'oggi non si sa quale sia stato il loro destino, se siano stati razziati dalle SS o dall'ERR, se bruciati durante i bombardamenti alleati su Francoforte o se requisiti dai soldati russi; solo una parte di quelli del Collegio rabbinico sono stati ritrovati e recuperati nel dopoguerra[26].

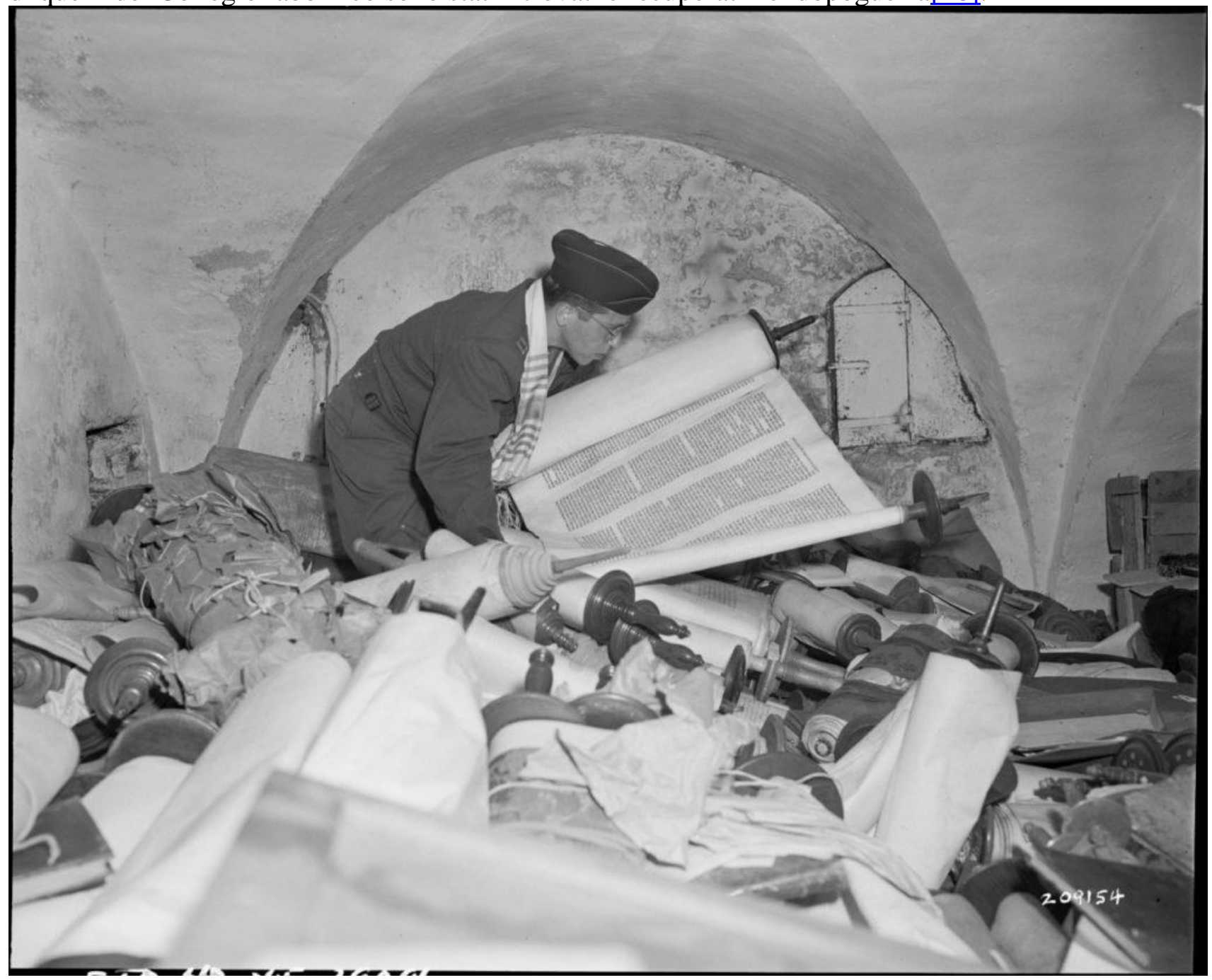

Fig. 9 Il cappellano militare Samuel Blinder nei sotterranei dell'ERR a Francoforte esamina un rotolo della Torah, tra le centinaia di quelli saccheggiati dai nazisti nei Paesi europei occupati, 6 luglio 1945 (National Archives, Stati Uniti, Archives.gov, Id. RG 111-SC-209154)

\section{Protezione, distruzione, ritrovamenti}

In attesa di essere smistati o di trovare collocazione dopo la guerra nei nuovi musei e biblioteche nazisti, i beni confiscati venivano stoccati in depositi: nello Jeu de Paume a Parigi, in castelli, come quello di Neuschwanstein in Baviera, in alcuni monasteri. Tuttavia, con l'intensificarsi dei bombardamenti alleati si temeva che questi edifici venissero danneggiati, come i musei e le chiese tedesche che contenevano, a loro volta, altre opere: a partire dal 1943 per accogliere tutti questi oggetti d'arte vennero allestite delle strutture di stoccaggio all'interno di miniere, come quelle di sale ad Altaussee in Austria. Nel giugno 1945 vi furono ritrovati 6577 dipinti, 230 disegni e acquarelli, 954 opere grafiche, 137 sculture, come pure arredi, armi e armature, arazzi, collezioni di libri[27]. Altri beni vennero depositati nelle miniere di Merkers, in Turingia, e Siegen, in Renania Settentrionale-Vestfalia. Nell'aprile del 1945 l'avanzata degli Alleati e dell'Armata rossa portò al ritrovamento di diversi di questi depositi, contenenti opere confiscate, opere provenienti da musei e chiese tedeschi, lingotti d'oro, valuta, beni preziosi. A guerra finita furono attivati processi di attribuzione e restituzione dei beni razziati, ma, nello stesso tempo, vi furono anche delle requisizioni da parte degli eserciti vincitori: si pensi ad esempio al caso della Madonna Sistina di 
Raffaello, incamerata dai russi come riparazione dei danni di guerra, restituita alla Pinacoteca di Dresda nel 1955 dopo un accordo tra URSS e DDR[28]. Già nel 1944, dopo la liberazione della Francia, gli Alleati avevano creato l'Art Looting Investigation Unit, il cui lavoro si intensificò dalla primavera del 1945. I beni ritrovati nelle miniere confluirono in alcuni punti di raccolta coordinati dal programma Monuments, Fine Arts, and Archives (MFAA); il più importante era il Central Collecting Point (CCP) di Monaco di Baviera[29]: qui venne incamerata e studiata la documentazione prodotta dalle varie agenzie e strutture naziste preposte alle spoliazioni (e spesso in competizione tra loro), furono effettuati moltissimi interrogatori, che ancora oggi costituiscono le fonti fondamentali per la Provenienzforschung, la "ricerca sulla provenienza". L'unità fu operativa fino all'inizio degli anni Cinquanta: i beni vennero il più possibile restituiti ai Paesi di provenienza, tuttavia spesso non fu possibile identificare i proprietari o ritrovarne gli eredi[30]. 


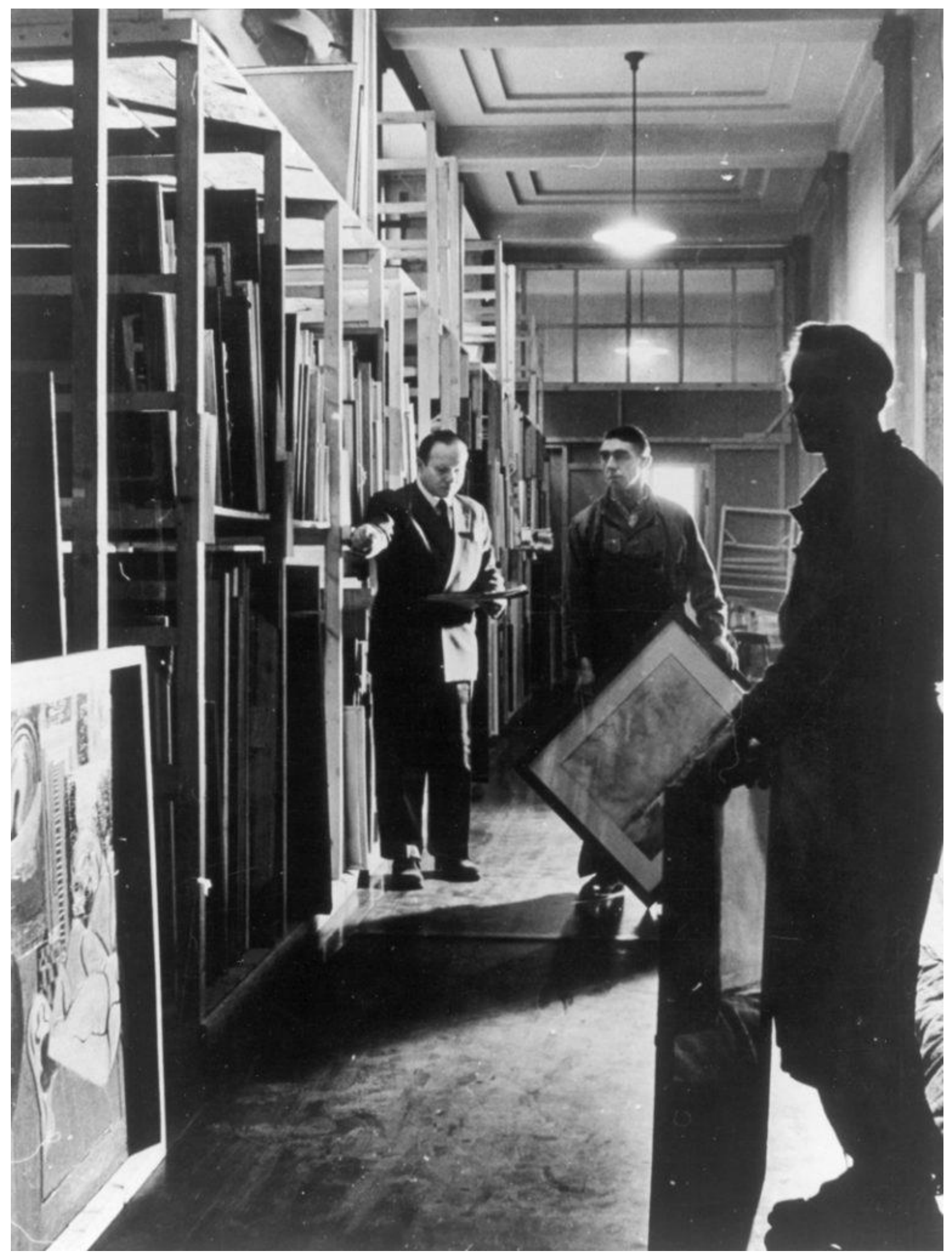

Fig. 10 Dipinti nei depositi del Central Collecting Point di Monaco, ca. 1945-1949 (foto: Johannes Felbermeyer. The Getty Research Institute, Los Angeles) 
Questa prima fase di restituzioni interessò in particolare le istituzioni pubbliche dei Paesi occupati: molto più complesso era ricostruire le provenienze dalle collezioni private $\mathrm{e}$, anche avendo identificato gli proprietari, trovarne gli eredi. La prassi prevedeva dunque di restituire le opere ai Paesi di provenienza degli ultimi proprietari, anche laddove vi fosse stata una connivenza di governi e amministrazioni collaborazionisti in quegli stessi Stati (si pensi al caso dell'Austria). A complicare ulteriormente il quadro vi era la difficile questione dei "metodi di acquisizione" attuati dai nazisti: vendite obbligate o gestione tramite tutori legali dei staatsfeindlisches Vermögen, $\mathrm{i}$ patrimoni dei "nemici dello Stato". Negli anni Novanta, con le trasformazioni europee succedute alla caduta del muro di Berlino, gli Stati coinvolti iniziarono a istituire commissioni per la restituzione delle opere razziate. $\mathrm{E}$ a permettere una maggiore trasparenza, consentendo di consultare gli archivi. Nel 1998, durante la Washington Conference on Holocaust Era Assets, vennero approvati anche i Washington principles on Nazi-Confiscated Art: partecipanti da 44 Paesi e associazioni di vittime sostennero la necessità di individuare le opere confiscate dai nazisti e restituirle agli eredi dei proprietari, promuovendo, in caso di contenziosi, soluzioni "fair and just"'[31]. In diversi casi gli eredi hanno avviato cause legali internazionali: controversie spesso dipanate sull'arco di anni, se non decenni, per la difficoltà di dimostrare che le opere erano state acquisite tramite minacce o illegalmente. Nel caso, invece, di acquisizioni postbelliche si rendeva necessario accertare se queste erano avvenute nella consapevolezza di essere di fronte al frutto delle razzie naziste[32]. A volte sono riemersi dal passato anche protagonisti delle spoliazioni: è il caso del ritrovamento del dipinto Le Quai Malaquais et l'institut, Printemps di Camille Pissarro, che negli anni Trenta apparteneva all'editore Samuel Fischer. Essendo ebrei, i Fischer fuggirono prima in Austria e poi, dopo l'Anschluss, verso gli Stati Uniti. Tutti i loro beni vennero sequestrati dalla Gestapo[33]: la loro collezione d'arte, tra cui molte opere degli impressionisti - considerati “degenerati” dall'estetica del regime - fu messa all'asta a Vienna nel 1940. Nel dopoguerra Gottifried Bermann Fischer, e successivamente sua figlia Gisela, cercarono di recuperare le opere della collezione fino a quando vennero contattati, tramite un intermediario, da un collezionista svizzero che avrebbe acconsentito alla restituzione del Pissarro. Emerse tuttavia che questo misterioso proprietario era in realtà Bruno Lohse, l'ex membro dell'ERR che nel dopoguerra aveva continuato a esercitare il mestiere di mercante d'arte, continuando a vendere tramite la sua società basata tra Svizzera e Liechtenstein opere di "dubbia" provenienza[34].

\section{Database e Provenienzforschung}

In parallelo alle complicate controversie legali, a partire dagli anni Novanta diverse istituzioni musei e centri di ricerca - hanno iniziato a compilare e successivamente mettere on line database e progetti sulla NS-Raubkunst: l'arte rubata dai nazisti. Il Deutsches Historisches Museum ha censito i dipinti confiscati dalla Sonderauftrag Linz (http://www.dhm.de/datenbank/linzdb/indexe.html), mentre la Frei Universität di Berlino ha catalogato le opere epurate dai musei tedeschi e confluiti nella mostra Entartete Kunst del 1937 (https://www.geschkult.fu-berlin.de/e/db_entart_kunst). Esistono poi dei database nazionali: l'Art Database of the National Fund of the Republic of Austria for Victims of National Socialism (https://www.kunstdatenbank.at/home.html); mentre il catalogo "Musées Nationaux Récupération" (http://www2.culture.gouv.fr/documentation/mnr/MnRpres.htm) raccoglie le opere restituite alla Francia ma per le quali non è stato ancora possibile individuare la provenienza privata, anche dopo i lavori dei tardi anni Novanta della Commissione Matteoli[35]. Diverse iniziative sono partite dagli Stati Uniti: molti documenti sulla spoliazione nazista sono infatti depositati presso i National Archives (https://www.archives.gov/publications/prologue/2002/summer/nazi-looted-art-1.html), in particolare alcuni album fotografici prodotti dall'ERR (https://www.archives.gov/research/holocaust/international-resources/nara/err) più recentemente, è stato messo in rete un database che raccoglie diverse ricerche sull'ERR supportate dall'United States Holocaust Memorial Museum (https://www.errproject.org/). Il Getty Institute di Los Angeles 
ha pubblicato diverse risorse on line sia per la ricerca delle provenienze, sia materiali di approfondimento sui beni ebraici razziati: si segnala in particolare il database sulle opere messe all'asta tra il 1930 e il 1945 in Germania, Austria, nei territori occupati dal Terzo Reich e in Svizzera (http://www.getty.edu/research/tools/provenance/german_sales.html) Per assistere gli eredi nelle ricerche, nel 1999 è stato creata la Commission for Looted Art in Europe (CLAE https://www.lootedartcommission.com) formata da storici dell'arte, storici e avvocati, in rappresentanza dell'European Council of Jewish Communities (ECJC) e della Conference of European Rabbis (CER), dal cui lavoro è scaturito The Central Registry of Information on Looted Cultural Property 1933-1945 (https://www.lootedart.com/home). Nel 2015 in Germania è stata istituita la Deutsches Zentrum Kulturgutverluste/German Lost Art Foundation (https://www.kulturgutverluste.de/Webs/EN/Start/Index.html), iniziativa governativa per cercare e identificare $i$ beni sequestrati dal regime nazista; anche in questo caso è stato creato un database (http://www.lostart.de/Webs/DE/LostArt/Index.html).

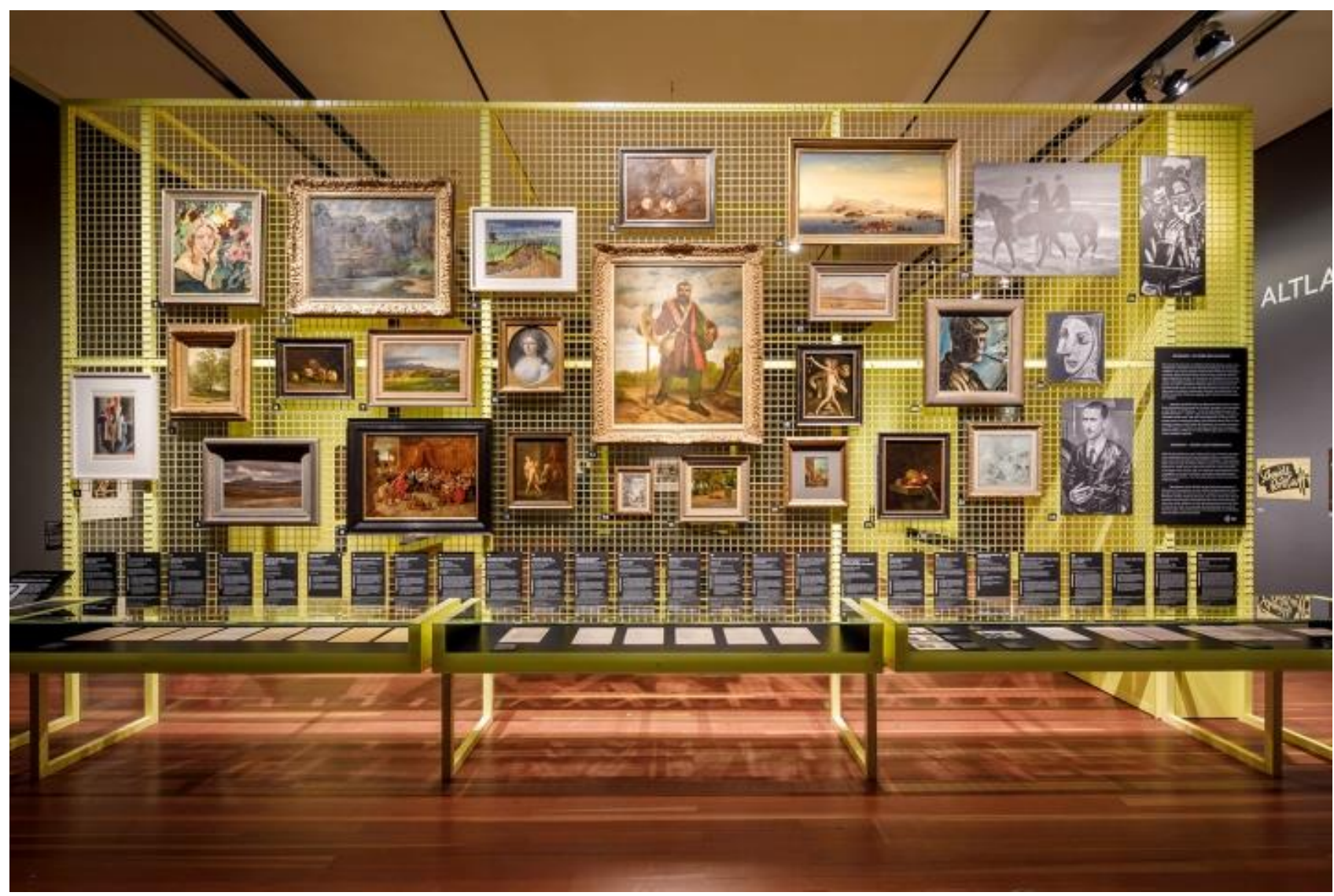

Fig. 11 Una sala della mostra Gurlitt: Status Report, An Art Dealer in Nazi Germany (foto: Bernd Lammel, 2018 @ Kunst- und Ausstellungshalle der Bundesrepublik Deutschland, Bonn)

\section{Lo Schwabinger Kunstfund, ovvero il caso Gurlitt}

Nel settembre 2010 sul treno tra Zurigo e Monaco venne fermato per un controllo un uomo anziano, Cornelius Gurlitt, che risultò in possesso di un ingente quantitativo di denaro contante. Scattarono una serie di accertamenti per supposta evasione fiscale (ipotesi che si rivelò infondata) che nel 2012 portarono a scoprire come nelle sue due abitazioni, a Monaco e a Salisburgo, fossero stivate circa 1.400 opere d'arte, in particolare dipinti e opere grafiche. Cornelius Gurlitt aveva ereditato questo patrimonio dal padre Hildebrand, storico e critico d'arte, nato in una famiglia di artisti, estimatore in particolare delle avanguardie dell'inizio del Novecento: sua sorella Cornelia era stata a sua volta una esponente dell'espressionismo negli anni attorno alla prima guerra mondiale. Con l'ascesa del nazismo, Hildebrand Gurlitt aveva perso il suo ruolo di direzione del Kunstverein di Amburgo e, 
come molti altri, aveva trovato fortuna come intermediario in quel mercato dell'arte ricco, spregiudicato, doloroso che si aprì tra la promulgazione delle leggi razziali e lo scoppio della guerra, rimanendo attivo fino agli ultimi mesi della stessa. Nel 2013 per via di un'inchiesta pubblicata sulla rivista "Focus", questo ritrovamento di opere d'arte - lo Schwabinger Kunstfund, dal nome del quartiere di Monaco dove si trovava la casa di Gurlitt - assunse una portata internazionale come scoperta di un "tesoro nazista" di altissimo valore. Tuttavia, alla luce del quadro ricostruito, a grandi linee, in questo articolo, il caso Gurlitt prende un carattere diverso da quello che gli hanno, con tono sensazionalistico, conferito i media. Hildebrand Gurlitt fu uno dei tanti, tantissimi, collaboratori del nazismo, in particolare all'interno dell'ampio e complicato piano di acquisizione e spoliazione delle opere d'arte europee: per gestire i rapporti tra venditori e acquirenti, per amministrare i patrimoni incamerati - ovvero espropriati - dei fuggitivi e dei deportati, fu necessario il lavoro di storici dell'arte, mercanti, avvocati. A spingere Gurlitt ad assumere il ruolo di mediatore nelle vendite e nelle confische di arte fu anche il suo essere considerato dalle leggi naziste Vierteljude, "ebreo per un quarto": meglio rendersi utile in questo settore caro a diverse figure preminenti del regime, che rischiare altre, ben peggiori, conseguenze. Nel dopoguerra venne interrogato dalla Art Looting Investigation Unit, che lo considerava uno dei principali mercanti d'arte assoldati dal regime nazista: tuttavia, Gurlitt, anche a causa della sua lontana origine ebraica e del suo lavoro di cura delle avanguardie, riuscì a presentarsi piuttosto come un difensore dell'arte. Negli anni Cinquanta, nominato direttore del Kunstverain di Düsseldorf, organizzò diverse mostre a supporto di questa interpretazione della sua attività: celando il proprio lucro, si mostrò come paladino della salvaguardia. Nel 2013 la "collezione Gurlitt" di Monaco venne sequestrata dallo Stato per effettuare controlli sulla provenienza: gli studi sono arrivati a definire che si trattava di opere, soprattutto delle avanguardie novecentesche "degenerate", che Gurlitt aveva o incamerato dalle epurazioni dei musei tedeschi, o acquistato da collezionisti ebrei (probabilmente a prezzi stracciati) per conto del regime, in modo da rivenderli all'estero e ottenere valuta straniera. In queste compravendite l'intermediario tratteneva una percentuale e, se le vendite non andavano a buon fine, le opere rimanevano in suo possesso, costituendo così la sua collezione. La qualità della raccolta di Gurlitt, di molto ridimensionata rispetto a quanto stimato inizialmente, testimoniava questa probabile casualità della sua formazione: un misto di opere di famiglia e del deposito dell'attività commerciale[36]. L'effetto positivo del caso Gurlitt è stato quello di dare nuova attenzione al tema delle opere d'arte razziate e alla ricerca delle provenienze, volta alla restituzione agli eredi. Con un colpo di scena, deludendo i suoi stessi eredi, è stato proprio Cornelius Gurlitt a indicare questa via, lasciando nel 2014 la sua collezione in eredità al Kunstmuseum di Berna, che ha scelto di accettare solo le opere di cui non è stata, ad oggi, ravvisata nessuna provenienza dalle razzie naziste nei confronti di collezionisti ebrei, e garantendo la restituzione agli eredi nel caso che questa eventualità venisse invece dimostrata. Le opere dalla provenienza più dubbia sono invece rimaste in Germania, oggetto del lavoro di un gruppo di ricerca: fino a ora sono state restituite poche opere, tra queste Femme Assise di Henri Matisse agli eredi di Paul Rosenberg e Zwei Reiter am Strand di Max Liebermann a quelli di David Friedmann.

\section{La riflessione artistica sulla spoliazione dell'arte}

Anche una semplice rassegna come quella effettuata in questo articolo mette in luce la dimensione capillare e vertiginosa dei progetti nazisti di spoliazione di opere, libri, oggetti, attraverso tutto il corpo europeo. Diversi sono gli artisti e autori contemporanei colpiti da questo aspetto del nazismo, che sembra essere come una sineddoche di quanto, in parallelo, accadeva agli uomini, spesso ai proprietari di quegli stessi beni. Dietro a ogni oggetto confiscato, stoccato, smistato, ci sono le persone a cui è appartenuto, a loro volta imprigionate, concentrate, deportate. Nel 2001 lo scrittore tedesco W.G. Sebald evocò in alcune pagine del suo romanzo Austerlitz una serie di immagini depositate al Bundesarchiv di Koblenz: si tratta di 88 fotografie in cui vengono mostrati momenti diversi della Möbel Aktion compiuta dall'ERR a Parigi. Sebald, che ha introdotto nella sua scelta 
stilistica l'uso delle immagini come elementi della narrazione, in questo caso, tuttavia, si limita invece alla descrizione, all'evocazione dei depositi della Gare d'Austerlitz, senza, stranamente, neppure citare l'album di Koblenz.

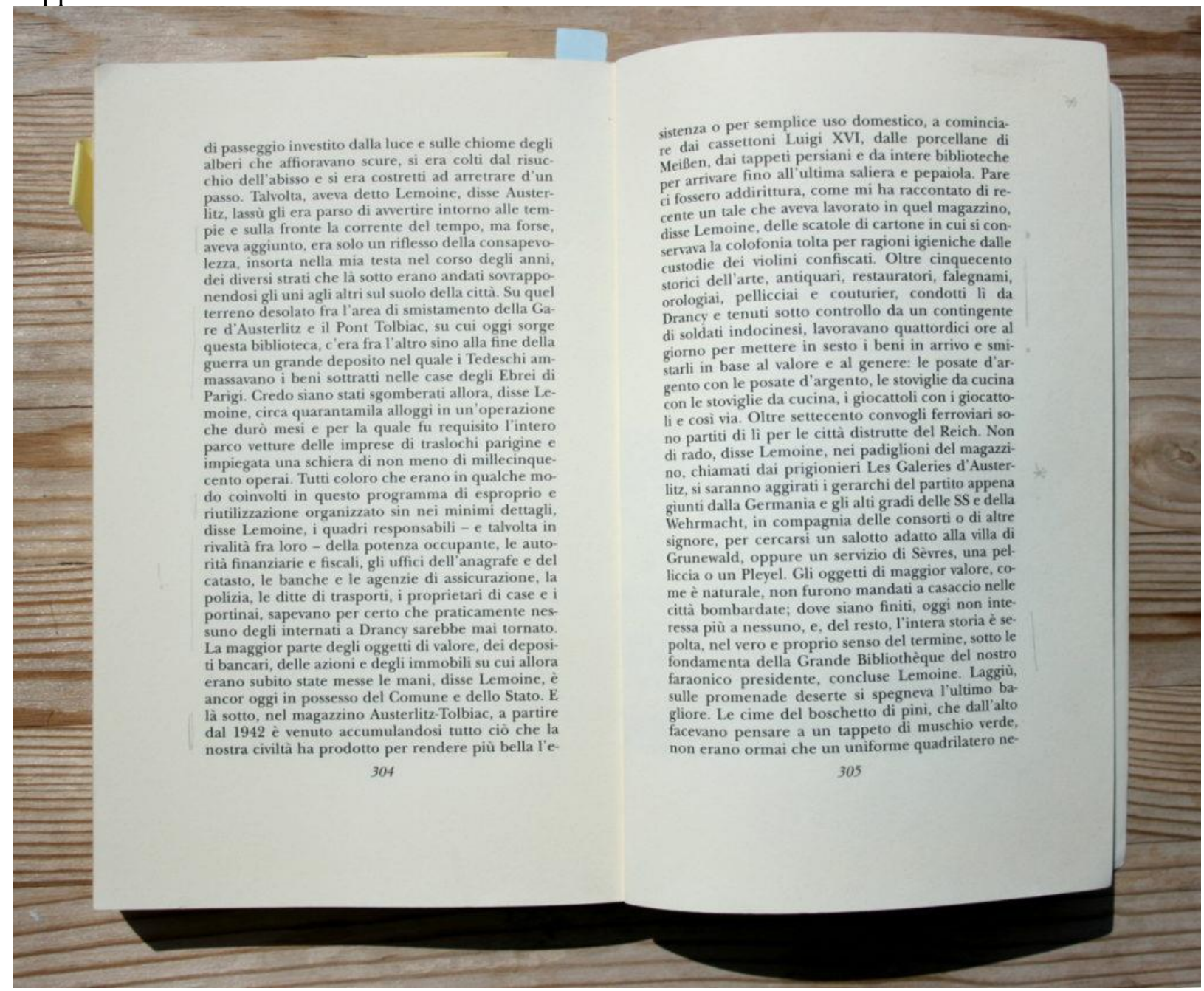

Fig. 12 W.G. Sebald, Austerlitz, Adelphi, Milano 2002

Nell'estate 2017 l'album sulla Möbel Aktion dell'ERR è stato esposto alla 14. edizione di documenta a Kassel, all'interno del progetto dell'artista tedesca Maria Eichhorn, che da diversi anni lavora sulla NS-Raubkunst, tramite il progetto Rose Valland Institute

(http://www.rosevallandinstitut.org). Al centro della sala dedicata al suo lavoro nel Fridericianum di Kassel, Eichhorn ha collocato una torre di libri: volumi conservati nella Zentral- und Landesbibliothek di Berlino, tutti inventariati con un numero progressivo e una J, quella di Jude, "ebreo". Si trattava di 2000 degli oltre 40.000 libri comuni (quelli di pregio avevano altre destinazioni) prelevati dalle biblioteche private degli ebrei "evacuati", tipico eufemismo della burocrazia nazista. E con un nuovo eufemismo, nel dopoguerra vennero rubricati come Geschenke, "donazioni", quando invece erano frutto della spoliazione sistematica di ogni bene degli ebrei della città. A partire da dediche ed ex libris, Maria Eichhorn, insieme al personale della biblioteca, sta cercando ora di risalire ai legittimi proprietari o ai loro eredi ma, laddove anche si riescono a ritrovare i nomi di queste persone, il loro destino è quasi sempre lo stesso: la morte nei campi di sterminio[37]. E forse è proprio per questo che Sebald decise di non mostrare quelle fotografie, ma di lasciare che le immagini si formassero nelle menti dei lettori. Immagini interiori, sintesi di innumerevoli storie, oggetti, uomini. 


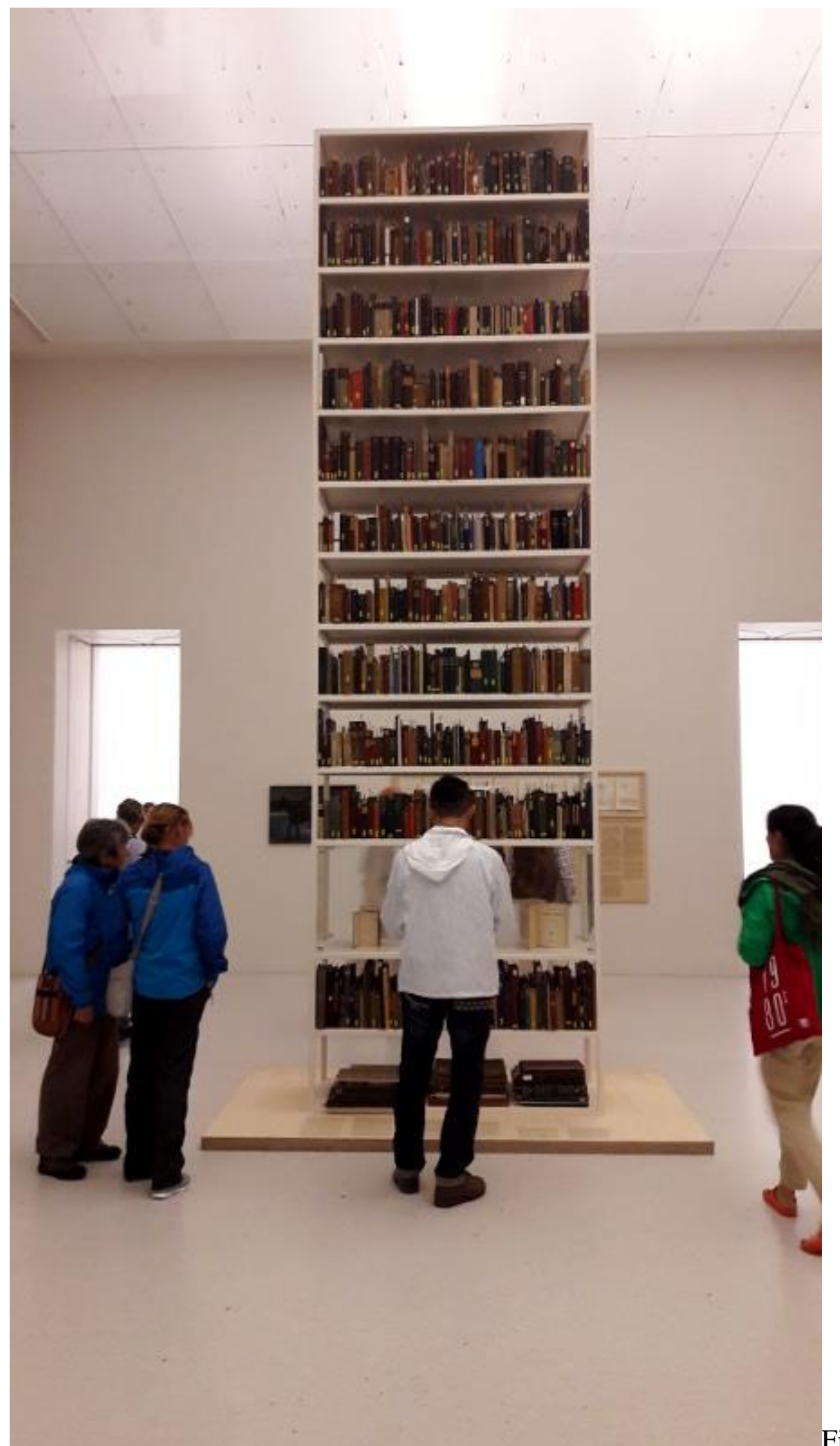

Fig. 13 Maria Eichhorn,

Unlawfully Acquired Books from Jewish Ownership by the Berliner Stadtbibliothek in 1943, registered in Zugangsbuch J (accession book J), documenta 14, Kassel, 2017 (foto: Elena Pirazzoli) 
[1] Otto Freundlich (1878-1973) venne imprigionato prima a Gurs, in Francia, poi venne deportato nel campo di concentramento e sterminio di Majdanek, vicino a Lublino, dove fu assassinato nel 1943.

[2] Il termine riprendeva le teorie di Max Nordau, seguace delle teorie di Cesare Lombroso e autore nel 1892 del volume Entartung, "degenerazione", in cui veniva tracciata una connessione tra la degenerazione delle forme artistiche e l'involuzione dei tratti somatici degli artisti. Il nazismo estese questa concezione, assumendo la prospettiva razzista e bollando come degenerate anche le opere realizzate da artisti ebrei: per paradosso, questa interpretazione avrebbe travolto lo stesso Nordau, ebreo.

[3] Sull'arte tedesca all'epoca del nazismo si veda Berthold Hinz, L'arte del nazismo, ed. orig. 1974, Mazzotta, Milano 1975.

[4] Si veda Ines Schlenker, Die "Grossen Deutschen Kunstausstellungen" und ihre Auswikungen auf den nationalsozialistischen Kunstbetrieb, in Kunst und Propaganda im Streit der Nationen 1930-1945, Hans-Jörg Czech, Nikola Doll (hrsg.), catalogo della mostra, Deutsches Historisches Museum Berlin 26.01-29.04.2007, Dresden, Sandstein 2007, pp. 258-267.

[5] Hans Belting, I tedeschi e la loro arte. Un'eredità difficile, ed. orig. 1992, Il Castoro, Milano 2005 , p. 46. Belting sottolinea anche come il contrasto all'arte moderna fosse presente nel dibattito sull'arte tedesca già prima dell'ascesa del nazismo: gli esponenti del regime utilizzarono gli elementi della discussione portata avanti in seno all'ambiente storico artistico già nei decenni prima (in particolare, le posizioni espresse da Henry Tode) per indirizzare l'opinione pubblica contro l'arte moderna, considerata internazionalista e, di conseguenza, antitedesca. In realtà, l'espressionismo proprio in quegli anni si stava configurando come "nuova arte tedesca" (Belting, $I$ tedeschi e la loro arte, cit., pp. 46-52).

[6] Thomas Mann, Fratello Hitler e altri scritti sulla questione ebraica, ed. orig. 1993, Mondadori, Milano 2005. L'articolo orginale è del 1939, come la poesia di Brecht Schlechte Zeit für Lyrik ("Tempi brutti per la poesia").

[7] Frederic Spotts, Hitler e il potere dell'estetica, ed. orig. 2002, Johan \& Levi, Milano 2012.

[8] Albert Speer, Memorie del Terzo Reich, Mondadori, ed. orig. 1969, Mondadori, Milano 1971; Elena Pirazzoli, Disumana e quotidiana. La scala monumentale del Nazismo, in Memorie di pietra. I monumenti delle dittature, a cura di Gian Piero Piretto, Raffaello Cortina, Milano 2014, pp. 117136.

[9] Le opere sul territorio italiano vennero coinvolte già prima grazie all'accondiscendenza di Mussolini che approvò vendite e doni personali a Hitler. In particolare il Führer reclamava i beni "tedeschi" dell'Alto Adige, dove i suoi collaboratori esperti d'arte speravano di trovare opere degli "antichi maestri" nordici, ma i funzionari italiani ostacolarono il loro trasferimento. Anche durante l'occupazione tedesca molte opere furono nascoste e salvate grazie all'intervento tempestivo di soprintendenti, funzionari, direttori di musei. Si veda: Ilaria Dagnini Brey, Salvate Venere! La storia sconosciuta dei soldati alleati che salvarono le opere d'arte italiane nella seconda guerra mondiale, Mondadori, Milano 2010; Paola Guidi, Uomini e tecnologie per la protezione dei beni culturali, Fondazione Enzo Hruby, Milano 2012; Salvatore Giannella, Operazione salvataggio. Gli eroi sconosciuti che hanno salvato l'arte dalle guerre, Chiarelettere, Milano 2014. La figura più nota è quella di Rodolfo Siviero, storico dell'arte e agente segreto: si veda Rodolfo Siviero, $L$ 'arte $e$ il nazismo. Esodo e ritorno delle opere d'arte italiane 1938-1963, Cantini Edizioni d'Arte, Firenze 
1984; Francesca Bottari, Rodolfo Siviero: avventure e recuperi del più grande agente segreto dell'arte, Castelvecchi, Roma 2013; Luca Scarlini, Siviero contro Hitler. La battaglia per l'arte, Skira, Milano 2014. Sul Kunstschutz si veda: Ernst Kubin, Raub oder Schutz? Der deutsche militarische Kunstschutz in Italien, Stocke, Graz 1994; Kunsthistoriker im Krieg. Deutscher Militärischer Kunstschutz in Italien 1943-1945, Christian Fuhrmeister C. (hrsg.), Böhlau, Köln 2012.

[10] Anche molte altre figure di spicco del nazismo parteciparono a questo spregiudicato mercato di opere d'arte, ad esempio il fotografo ufficiale di Hitler, Heinrich Hoffmann.

[11] Si veda Lynn H. Nicholas, The Rape of Europe. The Fate of Europe's Treasures in the Third Reich and the Second World War, Alfred A. Knopf, New York 1995, pp. 108-109.

[12] Troost morì nel 1934, da quel momento emerse la nota figura di Albert Speer, anche se non va dimenticato come furono diversi gli architetti coinvolti da Hitler direttamente per i suoi progetti più importanti. Tra questi, in particolare Hermann Giesler, cui venne ad esempio commissionato il progetto di revisione monumentale di Linz.

[13] Spotts, Hitler e il potere dell'estetica, cit., pp. 199-200.

[14] Il rapporto tra Posse e il regime nazista è molto ambiguo e complicato: inizialmente mal visto per le sue acquisizioni di arte moderna e quindi degenerata (in particolare di opere degli impressionisti tedeschi) e addirittura oggetto di delazione di matrice razziale, in cui veniva accusato di avere ascendenza ebraica, venne prima escluso dall'incarico alla direzione della Pinacoteca di Dresda e poi reintegrato. Alla sua morte, nel 1934, Hitler nominò suo successore Hermann Voss, storico dell'arte esperto in particolare di pittura tedesca e italiana - molto apprezzate dal Führer ma tuttavia noto per le sue posizioni democratiche, cosmopolite, antinaziste: probabilmente questa nomina inaspettata avvenne per via di un suggerimento dato da Posse prima di morire. Si veda Spotts, Hitler e il potere dell'estetica, cit., p. 201 e Birgit Schwarz, Hans Posse, Sonderbeauftragter für Linz, in "Frankfurter Allgemeine", 19 maggio 2000.

[15] Si veda Spotts, Hitler e il potere dell'estetica, cit., pp. 207-208 e Ernst Kubin, Sonderauftrag Linz: die Kunstsammlung Adolf Hitler. Aufbau, Vernichtungsplan, Rettung. Ein Thriller der Kulturgeschichte, Orac, Wien 1989.

[16] Walter Hofer (1893-1971 circa) non fu mai iscritto al partito nazista. Per conto di Göring comprò diverse opere nei paesi occupati, in particolare in Olanda, spesso battendo sul tempo gli uomini di Hitler. Dopo la guerra venne processato e condannato in contumacia a dieci anni, che però non scontò mai, continuando a esercitare il mestiere di mercante d'arte a Monaco fino alla sua morte.

[17] Si veda Les Archives diplomatiques \& Jean-Marc Dreyfus (a cura di), Le catalogue Goering, prefazione di Laurent Fabius, Flammarion, Paris 2015; si veda anche Arabella Cifani, Quel collezionista d'arte è un demonio, "Il Giornale dell'Arte", n. 363, aprile 2016, https://www.ilgiornaledellarte.com/articoli/2016/4/125998.html

[18] Bruno Lohse (1911-2007) nel dopoguerra, interrogato a lungo dagli Alleati, riuscì ad addossare tutte le colpe ai propri superiori presso l'ERR. Condannato a una breve detenzione e interdetto dalla professione di mercante d'arte, negli anni Cinquanta tuttavia riprese questa attività (si veda oltre). 
[19] Si veda Jonathan Petropoulos, Art As Politics in the Third Reich, University of North Carolina Press, 1999, p. 190.

[20] A Lucerna nel 1939 andarono all'asta diverse opere, tra cui un autoritratto di Van Gogh, si veda Nicholas, The Rape of Europe, cit. pp. 3-5.

[21] Valland inoltre segnalava alla resistenza francese quali erano i treni carichi di opere per evitare che venissero attaccati, danneggiano il prezioso carico. Allo stesso modo, dopo la liberazione di Parigi indicò agli Alleati le località tedesche in cui si trovavano i depositi per evitare che venissero bombardati. Si veda Rose Valland, Le front de l'art. Défense des collections françaises 1939-1945, Plon, Paris 1961; Les carnets de Rose Valland. Le pillage des collections privées d'oeuvres d'art en France durant la Seconde Guerre mondiale, sous la direction de Emmanuelle Polack et Philippe Dagen, Fage, Lyon 2011.

[22] La monumentale sede principale di questa "scuola di alti studi", disegnata dall'architetto Hermann Giesler, doveva venire realizzata sul lago di Chiemsee, in Baviera.

[23] A Praga avvenne un episodio grottesco. Già nel 1906 era stato creato un museo ebraico, ma dopo il 1939, quando Boemia e Moravia divennero protettorato tedesco, venne chiuso. Tuttavia, il personale del museo raccolse i beni di diverse comunità per proteggerli. Nel 1942 le SS assunsero e rovesciarono il significato di quella raccolta, creando lo Jüdisches Zentral Museum Prag, che era pensato come Museum einer untergegangenen Rasse, "museo di una razza estinta". Il personale del museo, espletati i lavori di catalogazione, venne inviato a Terezin. Si veda Jan Björn Potthast, Das jüdische Zentralmuseum der SS in Prag. Gegnerforschung und Völkermord im Nationalsozialismus, Campus, Frankfurt a.M.-New York 2002 e Il tesoro ebraico di Praga, Mondadori, Milano 1988, p. 11. Come scrive Hannah Arendt "[...] la creazione di musei che documentavano i loro nemici era una mania dei nazisti: durante la guerra vari organismi si disputarono l'onore di creare musei e biblioteche antiebraiche, e dobbiamo a questa singolare fissazione se tanti tesori della civiltà ebraica europea si sono salvati", in La banalità del male, ed. orig. 1963, Feltrinelli, Milano 1964, ed. cons. 1999, p. 45.

[24] Si veda Jean-Marc Dreyfus, Sarah Gensburger, Des camps dans Paris. Austerlitz, Lévitan, Bassano juillet 1943-août 1944, Fayard, Paris 2003; Sarah Gensburger, Images d'un pillage. Album de la spoliation des Juifs à Paris, 1940-1944, ÉditionsTextuel, Paris 2010.

[25] Johannes Pohl (1904-1960), prete cattolico, teologo, studioso di ebraismo, soggiornò a Roma e a Gerusalemme. Alla fine degli anni Trenta lasciò la Chiesa e nel 1940 entrò nel partito nazista, iniziando a lavorare per l'Institut zur Erforschung der Judenfrage e per l'ERR.

[26] Si veda il Rapporto sull'Attività della Commissione per il recupero del patrimonio bibliografico della Comunità ebraica di Roma, razziato nel 1943, 2009

(http://presidenza.governo.it/USRI/confessioni/rapporto/rapporto_finale_attivita_Commissione2.pd f); Ennio Matano, I predoni di Hitler e l'estinzione della memoria ebraica in Italia, "The Journal of Cultural Heritage Crime", 30.09.2018, https://journalchc.com/2018/09/30/la-memoria-della-culturaebraica-in-italia/

[27] Si veda Kubin, Sonderauftrag Linz, cit., p. 159.

[28] La questione delle requisizioni di opere effettuate dall'Armata rossa è ancora un problema aperto, si veda Konstantin Akinsha, Grigorii Kozlov, Sylvia Hochfield, Beautiful Loot. The Soviet Plunder of Europe's Art Treasures, Random House, New York 1995. 
[29] Gli archivi del Munich Central Collecting Point sono stati successivamente portati negli Stati Uniti e depositati prevalentemente presso il National Archives and Records Administration, una parte di fotografie e microfilm anche all'archivio fotografico del National Gallery of Art di Washington. Si veda Iris Lauterbach, The Central Collecting Point in Munich. A New Beginning for the Restitution and Protection of Art, Getty Publication, Los Angeles 2019.

[30] Il rapporto finale della American Commission for the Protection and Salvage of Artistic and Historic Monuments in War Areas, 1943-1946 può essere consultato qui:

https://www.fold3.com/title/759/roberts-commission-protection-of-historical-monuments

[31] Si veda https://www.state.gov/washington-conference-principles-on-nazi-confiscated-art/

[32] Per una rassegna di diversi casi di controversie legali, si veda Nicholas M. O'Donnell, A Tragic Fate: Law and Ethics in the Battle over Nazi-Looted Art, American Bar Association, Chicago 2017.

[33] Più precisamente dalla Vugesta, ovvero Verwertungsstelle für jüdisches Umzugsgut der Geheimen Staatspolizei, 1'“Ufficio per lo sfruttamento delle proprietà ebraiche della Polizia segreta di Stato".

[34] La vicenda ha altri risvolti significativi: a contattare Gisela Bermann Fischer furono Peter Griebert, mercante d'arte tedesco, e lo storico americano Jonathan Petropoulos, che chiesero una percentuale del valore dell'opera per il ruolo di intermediari. Tramite un'indagine, si scoprì che Griebert altro non era che un rappresentante della società Schönart di Bruno Lohse, la cui collezione si trovava depositata da decenni presso la Banca cantonale di Zurigo. Inoltre, il padre di Griebert era stato un collaboratore di Lohse durante le razzie naziste. Petropoulus sostenne di essere all'oscuro di tutto, e che la sua richiesta corrispondeva solo a un rimborso delle spese sostenute per le ricerche, ma scelse comunque di dimettersi dai suoi ruoli accademici. Si veda: Stefan Koldehoff, Pissarro Lost and Found, "ARTnews", 1 September 2007.

[35] Sugli esiti della Commissione Matteoli, si veda http://www2.culture.gouv.fr/documentation/mnr/MnR-matteoli.htm

[36] Per gli esiti della ricerca si veda:

https://www.kulturgutverluste.de/Webs/EN/ProjectGurlitt/Gurlitt-Provenance-Research/Index.html.

[37] Si veda: http://www.documenta14.de/en/artists/13489/maria-eichhorn; https://www.kulturgutverluste.de/Content/03_Forschungsfoerderung/Projekt/Zentral-undLandesbibliothek-Berlin/Projekt1.html 